\title{
Solid State Quantum Chemistry with $\Theta \Phi$ (ThetaPhi): Spin-Liquids, Superconductors and Magnetic Superstructures Made Computationally Available
}

\author{
A. Tchougréeff, E. Plekhanov† R. Dronskowski ${ }^{\ddagger}$ \\ January 28, 2021
}

\begin{abstract}
We present a standalone $\Theta \Phi$ (ThetaPhi) package capable to read the results of $a b$ initio DFT/PAW quantum-chemical solid-state calculations processed through various tools projecting them to the atomic basis states as an input and to perform on top of this an analysis of so derived electronic structure which includes (among other options) the possibility to obtain a superconducting (Bardeen-Cooper-Schrieffer, BCS), spin-liquid (resonating valence bond, RVB) states/phases as solutions of the electronic structure problem along with the magnetically ordered phases with an arbitrary pitch (magnetic superstructure) vector. Remarkably, different solutions of electronic-structure problems come out as temperature dependent (exemplified by various superconducting and spinliquid phases) which feature is as well implemented. All that is exemplified by model calculations on 1D chain, 2D square lattice as well as on more realistic superconducting doped graphene, magnetic phases of iron and spin-liquid and magnetically ordered states of a simplest nitrogen-based copper pseudo-oxide, $\mathrm{CuNCN}$, resembling socalled metal-oxide framework (MOF) phases by the atomic interlinkage.
\end{abstract}

Keywords: $\quad$ Superconducting phases, Spin-liquid phases, Spirally ordered magnetic phases, RVB, BCS, electronic structure of crystals.

*A.N. Frumkin Institute of Physical Chemistry and Electrochemistry of Russian Academy of Science, Moscow, Russia

†King's College London, Theory and Simulation of Condensed Matter (TSCM), The Strand, London WC2R 2LS, United Kingdom and A.N. Frumkin Institute of Physical Chemistry and Electrochemistry of Russian Academy of Science, Moscow, Russia

$\ddagger$ Institut für anorganische Chemie, RWTH Aachen University, Landoltweg 1, D-52056, Aachen, Germany; Jülich-Aachen Research Alliance, JARA-HPC, RWTH Aachen University, 52056 Aachen, Germany and Hoffmann Institute of Advanced Materials, Shenzhen Polytechnic, 7098 Liuxian Blvd, Nanshan District, Shenzhen, China 


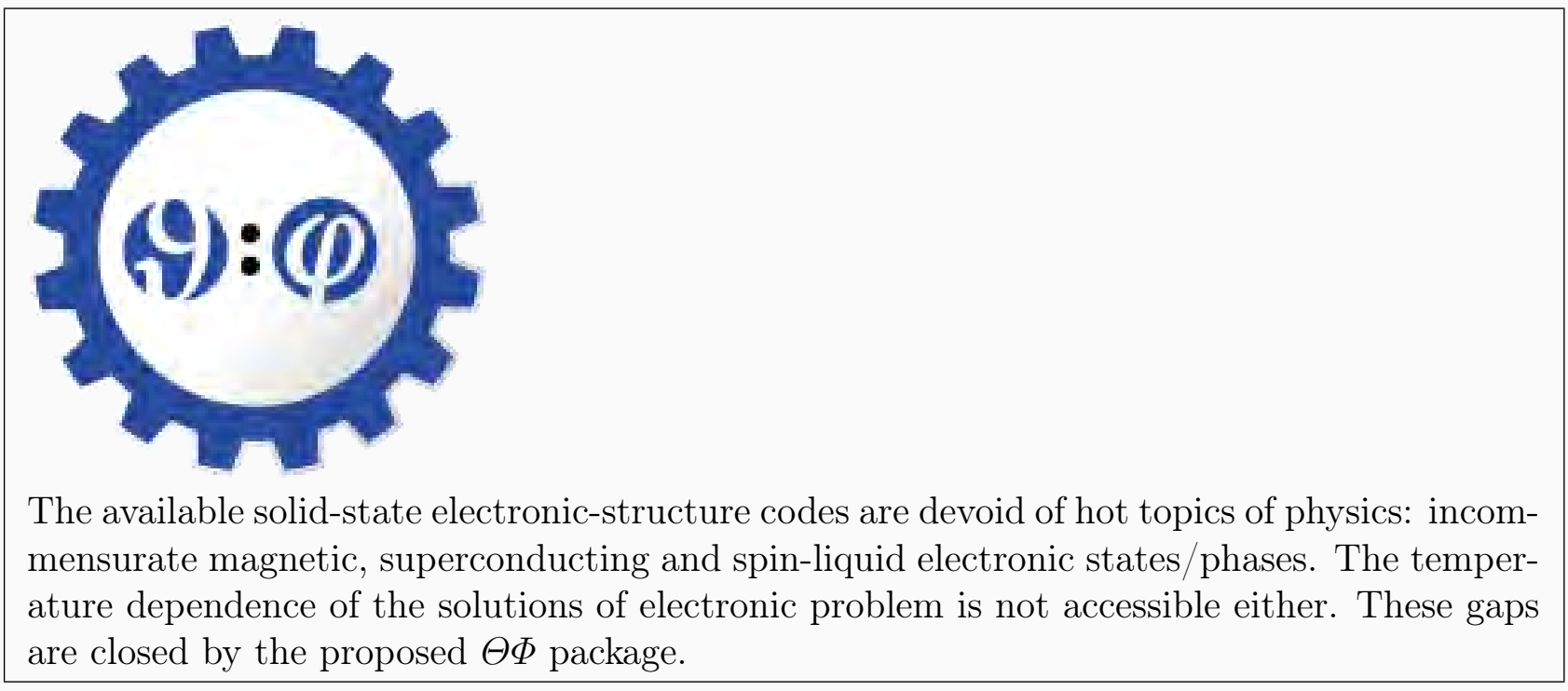




\section{INTRODUCTION}

Somewhat simplified, the presently available solid-state quantum-mechanical packages available are all based either on density-functional theory (DFT) for the electron-electron interactions or on the Hartree-Fock (HF) approximation for the electronic wave function. ${ }^{1}$ Given that the majority of materials is potentially metallic and taking into account the notorious problems of HF theory to deal with that, the overwhelming success of DFT for solid-state questions $^{2}$ is easily understandable, in particular when DFT is combined with plane waves and the pseudopotential/PAW approximations allowing to structurally optimize whatever kind of material based on exact Hellmann-Feynman forces. For certain nonmetallic materials, however, post-HF approaches are a powerful alternative, ${ }^{3}$ as are semi-empirical approximations to the HF approach for large systems. ${ }^{4}$ By this, the repertory of the types of the ground states accessible to the available software is significantly restricted. Practically, some of the important types of the electronic states of solids cannot be reproduced since they simply have not been programmed. The most striking (and scandalous) examples of inaccessible states are the Bardeen-Cooper-Schrieffer (BCS) state ${ }^{5,6}$ necessary for description of superconductors (SC) and the related resonating valence bonds (RVB) state introduced in the solid state context ${ }^{7}$ as an option for systems with frustrated antiferromagnetic interactions ultimately leading to the formation of spin-liquid phases. ${ }^{8,9}$ The RVB states have been

hypothesized to be those of high- $T_{c}$ cuprate superconductors. ${ }^{10}$ Even more familiar electronic states of solids - the magnetically ordered ones with an arbitrary pitch (superstructure) vector - are not easily accessible by the available numerical tools. The magnetically ordered states can be obtained by extending the chemical unit cells to super-cells and setting the primeval magnetic moments with broken symmetry (BS) in the input. Within this technology only the simplest ordered magnetic states, ferromagnetic or antiferromagnetic with very simple magnetic super-lattices, can be accessed. Thus, the presence of either BCS or RVB or complex magnetic states of solids is incurred indirectly. Namely, the presence of occupied anti-bonding one-electron states in the vicinity of the Fermi level and its intimate relationship with itinerant ferromagnetism was apparent when the bonding proclivities of metallic materials with strong exchange splitting were accessible from density-functional theory for 
the first time ${ }^{11,12}$ In addition, the presence of occupied non-bonding one-electron states as

found in, say, metallic bcc-Cr ${ }^{12,13}$ indicated a possibility of another kind of instability of the primary symmetric structure, namely the formation of an antiferromagnetic ground state. Although positioning the Fermi level in anti-bonding or non-bonding one-electron levels is a rational way to prepare ferromagnetic or antiferromagnetic metallic materials by design, ${ }^{14}$ it is not possible to predict what specific BS phase is going to appear. Moreover, the BS solutions of non-programmed types never come to surface in the calculations performed by standard tools and thus cannot be conjectured on the basis of numerical experiments. Another feature so far missing in the available software is the temperature dependence of the solutions of the electronic problems. This feature is, however, important due to characteristic physics: transitions among the high-temperature symmetric and various low-temperature BS phases are observed experimentally, the magnetic and superconducting phases being most spectacular. Thus, we recently undertook some developments with a goal to heal the outlined deficiencies in the existing software which resulted in the package $\Theta \Phi .{ }^{15,16}$ In previous works we implemented, respectively, (i) the BCS states and the magnetic phases with an arbitrary superstructure vectors ${ }^{15}$ and (ii) the energy optimization with respect to the vector of magnetic superstructure ${ }^{16}$ in the $\Theta \Phi$ package. By the present paper we round up the development of $\Theta \Phi$ by adding the option of having an RVB state (for more detailed explanation of these see below) as a result of the solution of an electronic problem. The paper is organized as follows: in the next Section we present the necessary theoretical concepts in a form more suitable for computational chemistry community in variance with papers ${ }^{15,16}$ more oriented towards the computational physics one; next we describe implementation details; furtheron we give test examples of applying developed software. Finally, we discuss the results of test calculations and give some perspectives.

\section{THEORY ACCOUNT}

Like in the standard approach, the $\Theta \Phi$ (Theta-Phi) program approximates the solution for the many-particle electronic Hamiltonian of a crystal. Its most general form admitted by $\Theta \Phi$ involves one-electron terms (the core attraction and hopping) and two-electron terms: 
that is one- and two-center Coulomb repulsion operators as well as the one- and two-center spin-spin exchange operators. Their explicit forms are available. ${ }^{15}$

Central objects in any mean-field theory are the mean-field version of the Hamiltonian, the Fockian, and the density matrix. In that or another form, they are present in all existing software. The electronic self-consistency problem is to be solved by requiring that a density matrix $\rho$ and the Hamiltonian define a Fockian $F[\rho]$, and the density stemming from its ground state has to be equal to that defining the Fockian:

$$
\rho \Leftrightarrow \rho(F[\rho])
$$

This is depicted in Figure 1. Hence, the derived density matrix approximates the ground state of electrons in a crystal, that is, its zero temperature phase.

In variance with the standard setting we want to be able to access different electronic

phases (magnetic, superconducting [alias BCS], spin-liquid [alias RVB]) which are temperature dependent. In order to address the temperature dependence one needs to minimize not the ground state energy, rather the Helmholtz free energy instead. Similarly, the additional phases (BCS, RVB, etc.) need to be included in the consideration. Thus, the general procedure of solving the electronic self-consistency problem Eq. (1) and Fig. 1 for a crystal needs to be modified at several points as described in the subsequent Subsections.

\section{Decoupling}

The first step of any mean-field theory is the decoupling serving to reduce the original many particle Hamiltonian to the mean-field Hamiltonian (Fockian) F. In the original Hamiltonian the electron-electron interactions (e.g. the Coulomb ones) enter through the products of four electronic creation and destruction operators $c_{1}^{\dagger} c_{2} c_{3}^{\dagger} c_{4}$. Based on Wick's theorem ${ }^{17}$ the products of four operators are replaced by an expression keeping products of only two operators complemented by the averages $\langle\ldots\rangle$ of the product of two remaining operators, by so called normal $\left(\left\langle c_{1}^{\dagger} c_{2}\right\rangle\right)$ and anomalous $\left(\left\langle c_{1}^{\dagger} c_{3}^{\dagger}\right\rangle\right.$ and $\left.\left\langle c_{4} c_{2}\right\rangle\right)$ elements of the 
density matrix:

$$
\begin{aligned}
c_{1}^{\dagger} c_{2} c_{3}^{\dagger} c_{4} & \Rightarrow\left\langle c_{1}^{\dagger} c_{2}\right\rangle c_{3}^{\dagger} c_{4}+c_{1}^{\dagger} c_{2}\left\langle c_{3}^{\dagger} c_{4}\right\rangle-\left\langle c_{1}^{\dagger} c_{2}\right\rangle\left\langle c_{3}^{\dagger} c_{4}\right\rangle \\
& -\left\langle c_{1}^{\dagger} c_{4}\right\rangle c_{3}^{\dagger} c_{2}-c_{1}^{\dagger} c_{4}\left\langle c_{3}^{\dagger} c_{2}\right\rangle+\left\langle c_{1}^{\dagger} c_{4}\right\rangle\left\langle c_{3}^{\dagger} c_{2}\right\rangle \\
& +\left\langle c_{1}^{\dagger} c_{3}^{\dagger}\right\rangle c_{4} c_{2}+c_{1}^{\dagger} c_{3}^{\dagger}\left\langle c_{4} c_{2}\right\rangle-\left\langle c_{1}^{\dagger} c_{3}^{\dagger}\right\rangle\left\langle c_{4} c_{2}\right\rangle .
\end{aligned}
$$

Note that the products of the two averages (the last terms in each row) are serving to make the mean value of the Fockian equal to the mean value of the original Hamiltonian calculated in the same approximation. In the standard software only the first two rows of Eq. (2) (habitually called direct or Hartree and exchange terms, respectively) are implemented, whereas the anomalous averages are forcedly set to zero. These averages are characteristic for the BCS and RVB solutions of the electronic structure problem, thus we retain them to be able to access the latter. The Fockian obtained through such decoupling is also known as the Fock-Bogolyubov operator. ${ }^{18}$ The non-vanishing anomalous averages allow us to break the symmetry tacitly implied in all quantum chemistry software: the conservation of the number of particles (electrons).

As usual we assume that each unit cell $r$ homes $L$ spatial states (atomic orbitals, AOs) numbered by subscript $l=1, \ldots, L$. For the sake of compactness of the subsequent presentation we assemble the Fermi operators corresponding to the spin projection $s=\downarrow$, $\uparrow$ in each of the AOs, pertaining to the unit cell $r$ of the solid, in a so called Nambu vector: ${ }^{19}$

$$
\Psi_{r}^{\dagger}=\left(c_{1 r \downarrow}^{\dagger}, c_{1 r \uparrow}^{\dagger}, \ldots, c_{L r \downarrow}^{\dagger}, c_{L r \uparrow}^{\dagger}, c_{1 r \downarrow}, c_{1 r \uparrow}, \ldots, c_{L r \downarrow}, c_{L r \uparrow}\right)
$$

It contains electron $c^{\dagger}$ and hole $c$ creation (electron destruction) operators and allows for uniform treatment of the normal, superconducting, spin-liquid and magnetically ordered states.

Since the anomalous averages are retained in Eq. (2) non-vanishing matrix elements between the electron $(e)$ and hole $(h)$ states appear in the Fockian operator as well, so that the latter acquires the block form:

$$
F=\left(\begin{array}{cc}
F_{e e} & F_{e h} \\
F_{h e} & F_{h h}
\end{array}\right)
$$

where, $F_{e h}=F_{h e}^{\dagger} ; F_{h h}=-F_{e e}$. The detailed forms of decoupled operators are available. ${ }^{15}$ 
In terms of the Fockian operator, the problem of minimizing the Helmholtz free energy reads:

$$
A=\langle F-\mu N\rangle-\theta \varsigma \rightarrow \min
$$

Here, $\langle\ldots\rangle$ is the mean value of the enclosed operator calculated with the density matrix $\rho$ : $\langle\ldots\rangle=\operatorname{Tr}(\ldots \rho) / \operatorname{Tr} \rho ; \varsigma$ is the Shannon entropy to appear here since the temperature $\theta=k_{B} T$ appears in the energy units. Since the Fockian contains the off-diagonal blocks mixing the electron and the hole states the quasi-particle states do not refer to any definite number of electrons. As a result the number of electrons $N$ is not conserved in the target BCS and RVB states and thus it is included in the optimization procedure accompanied by the chemical potential $\mu$ of electrons taking care about the conservation of their correct number on average.

The Helmholtz energy minimization then performs iteratively (as in the standard software), each iteration, as well as usual, divided in two stages: (i) the diagonalization of the operator $F-\mu N$ producing the spectrum of its quasi-particles; and (ii) the filling producing the new density $\rho^{\prime}$ which assures the minimum of the Helmholtz free energy for the spectrum of quasi-particles, obtained by the diagonalization. The obtained density $\rho^{\prime}$ is then reused for the decoupling of the original Hamiltonian and the iterations are repeated until the convergence in the density matrix and (free) energy is achieved.

\section{Diagonalization}

In terms of Nambu vectors Eq. (3) the Fockian operator rewrites:

$$
F=\sum_{r, \tau} \Psi_{r}^{\dagger} F(\tau) \Psi_{r+\tau}+\text { const }
$$

with the numerical constant so selected that $\langle F\rangle=\langle H\rangle^{15}$, and where $\Psi_{r}$ is a column, formed by the Fermi operators Hermitian conjugated to those in the row $\Psi_{r}^{\dagger}$. The summation extends to all unit cells $r$ and to lattice vectors $\tau$ of the relative shifts of the unit cells containing the states coupled through the matrices $F(\tau)$. It is translation invariant as it should be in a crystal. 
Diagonalization of the translationally invariant operators is habitually done as well in two steps: first, the transition from the basis of local atomic states to that of the Bloch sums (e.g. see $\left.^{1}\right)$ is performed. The translation invariant operator is block-diagonalized at this step: that is, reduces it to a (direct) sum of matrices numbered by the wave vectors $k$. Next, the $k$-numbered matrix blocks are diagonalized one-by one.

At this point the (block) diagonalization procedure is so modified that it opens access to electronic phases with arbitrary magnetic superstructure. In the latter the electronic magnetic momenta (if any) appearing in the unit cells shifted by a lattice vector $\tau$ are rotated relative to each other by an angle $(\tau, \mathbf{Q})$ around a unit vector $\mathbf{n}$, where $\mathbf{Q}$ is a superstructure (pitch) wave vector. Since either $\mathbf{n}$ or $\mathbf{Q}$ may be arbitrarily directed and even more $\mathbf{Q}$ may be arbitrarily small, the momenta in the neighbor unit cells may be relatively rotated by an arbitrarily small angle giving rise to arbitrary (magnetic) spiral states. This is reached by rotating the spin-quantization axes in each unit cell by the angles $\vartheta=(r, \mathbf{Q})$ around $\mathbf{n}$ and builing the (generalized) Bloch sums from the local states quantized relative these, unit cell specific, axes. ${ }^{20-22}$

Formally, we replace the usual numerical phase factors for the local states in the unit cell $r$ : $\exp (i k r)$ by $2 \times 2$ spin-rotation matrices $\exp (i k r) \Omega_{\mathbf{n}, \vartheta}$ transforming the one-electron states for the l-th AO with "up" and "down" spin projections relative to the laboratory $z$-axis to the similar ones, but with the spin projections relative to the unit cell specific $z$-axes. The matrices are given by:

$$
\Omega_{\mathbf{n}, \vartheta}=\sigma^{0} \cos \frac{\vartheta}{2}-i(\mathbf{n}, \boldsymbol{\sigma}) \sin \frac{\vartheta}{2},
$$

where $\boldsymbol{\sigma}=\left(\sigma^{x}, \sigma^{y}, \sigma^{z}\right)$ is the vector of Pauli matrices, while $\sigma^{0}$ is the $2 \times 2$ identity matrix. The matrices $\exp (i k r) \Omega_{\mathbf{n}, \vartheta}$ are applied to each of the pairs of operators $c_{l r \downarrow}, c_{l r \uparrow}$ in the Nambu vector (and their Hermitian conjugates to the respective $c_{l r \downarrow}^{\dagger}, c_{l r \uparrow}^{\dagger}$ pairs). Then, as usual, summation over $r$ is performed:

$$
\Psi_{k}=\frac{1}{\sqrt{\mathrm{K}}} \sum_{r} \exp (i k r) \Omega_{\mathbf{n},(r, \mathbf{Q})} \Psi_{r},
$$

where $\mathrm{K}$ is the number of the $k$-points involved in the calculation. This makes the Fockian 
operator block-diagonal with the blocks numbered by the wave vectors $k$ :

$$
\begin{array}{r}
F=\sum_{k} \Psi_{k}^{\dagger} F^{(k)} \Psi_{k}, \\
F^{(k)}=\sum_{\tau} e^{i k \tau} \Omega_{\mathbf{n},(\tau, \mathbf{Q})} F(\tau)
\end{array}
$$

(here we omitted the immaterial const which can be restored in the end to maintain the overall energy balance). Even in this case the dimension of the Fockian blocks $F^{(k)}$ remains the same: $4 L \times 4 L$ so that arbitrary magnetic superstructures can be addressed without increasing the size of the unit cell.

\section{Filling}

Lastly, the filling generating the new density matrix from the eigenvectors of the Fockian blocks $F^{(k)}$ is somewhat different from the usual filling of the particle states by electrons implemented in existing solid state packages. Namely, at this point the desired temperature dependence of electronic phases enters.

Indeed, diagonalization of $F^{(k)}$ 's yields its eigenvalues $\varepsilon_{\alpha, k} \alpha=1 \div 4 L$ and eigenvectors. The latter are assembled in unitary matrices $\Xi_{k}$. The matrix $\Xi_{k}$ when applied to the corresponding generalized Bloch sum of the Nambu vectors Eq. (8) produces the Nambu vector of canonical quasi-particles:

$$
B_{k}=\left(\beta_{1 k}, \beta_{2 k}, \ldots, \beta_{4 L k}\right)=\Xi_{k} \Psi_{k}
$$

As we already mentioned, the quasi-particles solving the energy minimum problem for the $F-\mu N$ operator are superpositions (linear combinations) of the electron and hole states as suggested by Bogolyubov, Tolmachev and Tyablikov yet more than 60 years ago. ${ }^{23-26}$ Thus they are referred to as bogolons in the BCS context. In their basis the sought density matrix is diagonal. The equilibrium populations of the quasi-particle states $\beta_{\alpha, k}$ assuring the free energy minimum for a given spectrum $\varepsilon_{\alpha, k}$ of quasi-particles ${ }^{18,27,28}$ is given by :

$$
\rho_{\alpha \alpha^{\prime}}^{(k)}=\delta_{\alpha, \alpha^{\prime}} f\left(\frac{\varepsilon_{\alpha, k}(\mu)}{\theta}\right)
$$

where $f(x)=\left(1+e^{x}\right)^{-1}$ - the Fermi-Dirac distribution. 
Since the number of electrons $n_{\alpha, k}$ in the quasi-particle states is not mandatorily equal to unity, in order to keep the average number of electrons in the unit cell equal to $N_{0}$ prescribed by the chemical composition:

$$
\frac{1}{\mathrm{~K}} \sum_{\alpha, k} f\left(\frac{\varepsilon_{\alpha, k}(\mu)}{\theta}\right) n_{\alpha, k}=\langle N\rangle=N_{0},
$$

the chemical potential $\mu$ of electrons is adjusted. As $\theta \rightarrow 0$ the Fermi distribution flows to the standard step-like one, whereas the chemical potential $\mu$ of electrons flows to the Fermi energy $\varepsilon_{F}$.

At the same time, it can be shown ${ }^{18}$ that the spectrum of bogolons $\varepsilon_{\alpha, k}$ obtained by diagonalization of the $F^{(k)}-\mu N$ blocks of the structure Eq. (4) is always symmetric with respect to zero; namely: for each quasi-particle state with positive energy $\varepsilon_{\alpha, k}$ there exists a quasi-particle state with the opposite energy $-\varepsilon_{\alpha, k}$ and vice versa, so that the chemical potential of quasiparticles equals to zero (as it is in Eq. (11)).

The density Eq. (11) is to be used in the decoupling of the interaction terms. For this end it must be back transformed to the representation of the original AOs, which is done easily with use of the matrices $\Xi_{k}$ :

$$
\left\langle\Psi_{r} \Psi_{r+\tau}^{\dagger}\right\rangle=\sum_{k} \exp (-i k \tau) \Omega_{\mathbf{n},(\tau, \mathbf{Q})}^{\dagger} \Xi_{k}^{\dagger} \rho^{(k)} \Xi_{k} .
$$

The result does not depend on $r$ because of the translational invariance (the expressions $\Psi_{r} \Psi_{r+\tau}^{\dagger}$ are themselves matrices filled by all possible products of the Fermi operators from the respective Nambu vectors). Because of the translational invariance, one can introduce equivalent $4 L \times 4 L$ matrix blocks:

$$
\rho(\tau)=\mathbf{1} \delta_{\tau, 0}-\left\langle\Psi_{r} \Psi_{r+\tau}^{\dagger}\right\rangle
$$

each accumulating matrix elements of the density between the states pertinent to the unit cells shifted by the lattice vectors $\tau$, while 1 means a $4 L \times 4 L$ identity matrix; $\rho(\tau=0)$ is Hermitian, while at a finite shift $\tau \neq 0$ the following relation holds:

$$
\rho(\tau)=\rho^{\dagger}(-\tau)
$$

i.e. Hermitian conjugate of a density matrix at the lattice shift $\tau$ is the density matrix at the lattice shift $-\tau$ assuring the hermiticity of the density matrix as a whole. 


\section{Physical properties}

Once self-consistency is reached, the observables can be calculated, that is correlation functions as well as band structure and density of electronic states (DOS). Below we summarize the formulas for some of them, ${ }^{15}$ but many others can be defined in addition. At the selfconsistency, the internal energy $U$ is just an average of the Fockian operator $F$ :

$$
U=\langle F\rangle=\frac{\operatorname{Tr}(F \rho)}{\operatorname{Tr} \rho}=\frac{1}{\mathrm{~K}} \sum_{\alpha, k} f\left(\frac{\varepsilon_{\alpha, k}}{\theta}\right) \varepsilon_{\alpha, k}
$$

(hereinafter we omit the $\mu$-dependence of the spectrum for the sake of brevity).

The (Helmholtz) free energy $A$ and the entropy $\varsigma$ Eq. (5) are defined by the spectrum of the canonical quasi-particles:

$$
\begin{gathered}
A=-\theta \sum_{\alpha, k} \ln \left(1+e^{-\varepsilon_{\alpha, k} / \theta}\right), \\
\varsigma=-\frac{\partial A}{\partial \theta}=\frac{U-A}{\theta}=-\sum_{\alpha, k}\left\{f\left(\frac{\varepsilon_{\alpha, k}}{\theta}\right) \ln f\left(\frac{\varepsilon_{\alpha, k}}{\theta}\right)+\left(1-f\left(\frac{\varepsilon_{\alpha, k}}{\theta}\right)\right) \ln \left(1-f\left(\frac{\varepsilon_{\alpha, k}}{\theta}\right)\right)\right\} .
\end{gathered}
$$

The expression Eq.(14) is used in practical calculations as anticipated above. The (electronic) specific heat can be easily obtained:

$$
C_{V} / k_{B}=\frac{\partial U}{\partial \theta}=-\theta \frac{\partial^{2} A}{\partial \theta^{2}}
$$

The uniform Pauli magnetic susceptibility is given by:

$$
\chi_{P}=\frac{\partial S_{t o t}^{z}}{\partial \mathcal{H}_{z}}
$$

where $S_{\text {tot }}^{z}$ is the total magnetic moment of the unit cell:

$$
S_{\text {tot }}^{z}=\sum_{l} S_{l, 0}^{z}=\frac{1}{2} \sum_{l}\left\{\left\langle c_{0 l \uparrow}^{\dagger} c_{0 l \uparrow}\right\rangle-\left\langle c_{0 l \downarrow}^{\dagger} c_{0 l \downarrow}\right\rangle\right\},
$$

expressed through the density matrix elements between the local states.

The band structure along the path connecting the high symmetry points in the Brillouin zone is obtained by plotting $\varepsilon_{\alpha, k}$ along it. Finally, the density of states is obtained from $\varepsilon_{\alpha, k}$ as usual:

$$
D(\epsilon)=\frac{1}{\mathrm{~K}} \sum_{\alpha, k} \delta\left(\epsilon-\varepsilon_{\alpha, k}\right)
$$


Closing the discussion of the observables, we note that the solutions of self-consistency equations have their domains of existence as functions of Hamiltonian parameters, filling, temperature and any other external condition. These domains form the system's phase diagrams, which can be directly compared with the experiment and/or other theories. As well, there might exist (and this is the standard situation) several solutions or phases for the same values of the Hamiltonian parameters, and/or temperature. In this case, owing to the variational principle, the phase with the lowest free energy must be chosen, which paves the way to description of the phase transitions at finite temperature.

\section{IMPLEMENTATION DETAILS}

\section{Self-consistency cycle}

The general (free) energy optimization procedure as applied to the electronic phases of a crystal can be solved in two ways:

1. either as a self-consistency procedure (as in details described above): starting from a trial $\rho$ and iteratively calculating new $\rho^{\prime}$ at each step, taking care about the chemical potential. In order to stabilize such a procedure usually some forms of mixing are used. We implemented the simplest linear mixing, such that $\rho \Leftarrow \alpha \rho^{\prime}+(1-\alpha) \rho$ with $0 \leqslant \alpha \leqslant 1$

or

2. by solving the self-consistency equation Eq. (1) supplemented with the constraint fixing the number of particles as an equation for roots using the globally convergent Newton-Raphson method ${ }^{29}$ with numerically estimated derivatives of the right hand side of Eq. (1). It can be shown that the self-consistency condition is equivalent to the evanescence of the derivatives of the energy with respect to relevant matrix elements of the density. The energy is traced at each step to ensure that the chosen trial step actually leads to a minimum.

Both approaches are implemented in $\Theta \Phi$. 
Of course, a self-consistency procedure can find an extreme of the free energy which is not necessary the (global) minimum. Therefore, we also track the values of the free energy as self-consistency proceeds and control that it always goes down.

In case of multi-orbital systems and complicated lattices the inter-relations between density matrix elements at various distances (see e.g. Ref. ${ }^{30}$ ) may become very involved. It is advisable to analyze them before using $\Theta \Phi$. We shall discuss this in more details in the Examples Section below.

\section{Matrix elements and porting with existing programs}

It is a common place to contrast model and quantum chemical ( $a b$ initio or semi-empirical) codes. Meanwhile, the difference is merely quantitative: codes of either type operate with matrices formed according to prescriptions of the Theory Section with the only difference that the model approaches use a small number of states in the unit cell (small number of bands) and with respective (Fockian) matrix elements dependent on relatively small number of model parameters, whereas quantum chemistry packages employ realistic number of atomic states and calculate the required matrix elements from the parameters of these latter or pseudopotentials. The $\Theta \Phi$ package bridges the model approach and quantum chemistry one by implementing the true multi-band scheme. As for the Hamiltonian/Fockian matrix elements, the user can either specify them all manually, assuming an abstract theoretical model, or alternatively use an additional functionality allowing the import of the hopping matrix elements $t_{l, l^{\prime}}^{s, s^{\prime}}(\tau)$ from existing $a b$ initio codes. This is achieved by reading the tight-binding Hamiltonians, generated by either of the two well-known codes: WANNIER $90^{31}$ and LOBSTER ${ }^{32-34}$ serving to post-process the outputs of the DFT/PAW calculations. By doing this, the precise, material-specific analysis of electronic phases of various crystals can be made. $\Theta \Phi$ reads the files wannier90_hr.dat (for WANNIER90) or RealSpaceHamiltonians.lobster (for LOBSTER) and populates the hopping matrix. Both WANNIER90 and LOBSTER have in turn interfaces to the major codes (like VASP, ABINIT, CASTEP, QUANTUMESPRESSO etc.), which allows the $\Theta \Phi$ user to profit from the results obtained by them. Additionally, we can recommend using the MAGAîxTIC package ${ }^{35}$ to obtain the estimates of the $J_{l, l^{\prime}}^{\alpha \beta}(\tau)$ - diatomic exchange - parameters. In the future releases we plan to provide a 
functionality of directly evaluting the Hamiltonian/Fockian matrix elements from chemical composition/crystal structure data as in all quantum chemistry codes.

\section{EXAMPLES}

The features of $\Theta \Phi$ described in the previous Sections has been implemented and tested ${ }^{15,16}$ against simple analytic models, the 1D Hubbard superconductor, 2D doped graphene superconductor and square lattice doped antiferromagnet as well as against realistic models of magnetic states of $\alpha$ - and $\gamma$-Fe. Below we shall briefly review these results before specially concentrating upon the newest feature implemented in $\Theta \Phi$ : spin-liquid (alias RVB) states as compared to the magnetically ordered states of $\mathrm{CuNCN}$.

\section{Superconducting and magnetic phases of Hubbard model}

The 1D single band Hubbard model is the simplest one for, say, electrons in polyene molecules $(\mathrm{CH})_{\mathrm{n}},{ }^{36}$ where each $\mathrm{C}$ atom holds one $\pi$-orbital or of individual chains of a metallic carbon allotrope hypothetized by Hoffmann et al. ${ }^{37}$ as depicted in Figure 2. In the model setting it is characterized by three parameters: inter-site (" $\pi-\pi ")$ one-electron hopping $t$, on-site electron-electron interaction $U$ and the average electron population per site $1-\eta$, where $\eta$ - here is referred to as a doping level. Accepting hereinafter $t=1$ to be the energy scale, the other model parameters as well as temperature express in its terms. Depending on the sign of $U$ either a magnetic $(U>0)$ or superconducting $(U<0)$ states come out as solutions of the mean-field electronic problem. The latter corresponds to the density matrix of the form:

$$
\rho=\left(\begin{array}{cccc}
n / 2 & 0 & 0 & -\Delta^{\star} \\
0 & n / 2 & \Delta^{\star} & 0 \\
0 & \Delta & 1-n / 2 & 0 \\
-\Delta & 0 & 0 & 1-n / 2
\end{array}\right) .
$$

The dimensionality of four is that of the subspace of quasipatricles which within the $\Theta \Phi$ setting stems from a single AO per unit cell by including two respective spin-projection states and two corresponding hole states. The components of the density matrix, thus, have 
the following meaning: $n=\left\langle c_{s}^{\dagger} c_{s}\right\rangle, \Delta=\left\langle c_{\uparrow}^{\dagger} c_{\downarrow}^{\dagger}\right\rangle$ both independent on the site index which is thus omitted for brevity.

The $\Theta \Phi$ results for this simple model are shown in Fig. 3. As expected from the BCS theory, the temperature dependence of the anomalous matrix element of the density $\Delta$ (the Figure 3 superconducting order parameter) is described with a very good accuracy by:

$$
\Delta(\theta)=\Delta_{0} \sqrt{1-\left(\frac{\theta}{\theta_{c}}\right)^{3}} .
$$

The temperature dependence of the solution of the electronic problem is a very remarkable feature provided by $\Theta \Phi$. Incidentally, the $U$-dependence of $\Delta_{0}$ at lowest temperature is very well described by another BCS formula:

$$
\Delta_{0}(U)=-\frac{8 e^{\frac{2 \pi}{U}}}{U}
$$

for small absolute values of negative i.e. attractive $U$ as well nicely numerically reproduced by $\Theta \Phi$.

Extending similar treatment to a square lattice of sites with the hopping $t$ between the nearest neighbors (a kind of model for one layer of simple cubic $\alpha$-Po with only $\pi$-orbitals included in the consideration) provides additional set of tests went through by $\Theta \Phi$. For the square lattice 2D Hubbard model we studied tentative magnetic states of this model $(U>0)$ under finite doping which may serve as a model of the corresponding simple cubic layer deposited over some support. The single band Hubbard model with magnetic order can be characterized by the density matrix of the form:

$$
\rho=\left(\begin{array}{cccc}
\frac{n-m}{2} & 0 & 0 & 0 \\
0 & \frac{n+m}{2} & 0 & 0 \\
0 & 0 & 1-\frac{n-m}{2} & 0 \\
0 & 0 & 0 & 1-\frac{n+m}{2}
\end{array}\right) .
$$

Here, as well, the dimensionality of four is that of the space of quasiparticles stemming from one AO per unit cell; in variance with $1 \mathrm{D}$ superconducting phase the anomalous averages occupying the (northeast) antidiagonal are vanishing; $n=1-\eta$ is the average site occupation, while $m$ is the average site magnetization. Although the matrix refers to the single 
unit cell (site) and describes the imbalance of the occupations of the states with different spin projection - seemingly of a ferromagnetic state - this imbalance takes place with respect to the adjustable local spin quantization axes as implemented in $\Theta \Phi$. Thus, depending on the pitch wave vector Q, Eq. (17) describes an arbitrary magnetically ordered state (e.g. $\mathbf{Q}=(\pi, \pi)$ results in the antiferromagnetic state) since the momenta of the magnitude $m$ are rotated by the angle $(\mathbf{Q}, \tau)$ relative to each other in the unit cells shifted by the translation vector $\tau$. The results of the $\Theta \Phi$ study of this model are depicted in Figure 4.

One can see that at the vanishing doping the Hubbard model treated by $\Theta \Phi$ consistently yields a checkerboard antiferromagnetic state with $\Delta Q=0$ provided the pitch vector is set to be

$$
a \mathbf{Q}=(\pi, \pi)+(\Delta Q, \Delta Q)
$$

( $a$ is the lattice constant) in a full agreement with the general theory and the numerical findings. ${ }^{21}$ At a moderate doping level of $\eta=0.15, \Theta \Phi$ produces an energy profile shown on the left of Figure 4 identical to that of Ref. ${ }^{21}$.

\section{Superconducting states of graphene}

Superconductivity in pristine graphene does not occur naturally, although recently the superconductivity has been reported in graphene bilayers rotated at a series of "magic" angles. ${ }^{38,39}$ Several approaches predicted superconductivity with various order parameter symmetries on 2D honeycomb lattice, among which auxiliary-boson theory $(\mathrm{ABF})^{30,40}$ or even self-consistent Bogoliubov-de Gennes approach to spatially inhomogeneous superconductivity. ${ }^{41,42}$ It is excessive to reiterate that neither of mentioned solutions of the electronic structure problem is accessible to the available solid state quantum chemistry codes.

We benchmarked $\Theta \Phi$ by reproducing several superconducting phases of various symmetries in graphene. We remind that on 2D honeycomb (graphene) lattice each lattice site connects to three nearest neighbor $(\mathrm{NN})$ sites and the unit cell of graphene contains two atoms (see Fig. 5). With only NN hopping active, each unit cell is connected to its four nearest neighbors at the lattice shifts $\tau= \pm a_{1}, \pm a_{2}$.

Superconducting phases on the honeycomb lattice with the interactions between the NN 
sites shifted by vectors $\delta_{i}$ feature the anomalous averages $\Delta_{\mathrm{AB}}\left(\delta_{i}\right)=\left\langle c_{\mathrm{A} r \uparrow}^{\dagger} c_{\mathrm{B} r+\delta_{i \downarrow} \downarrow}^{\dagger}\right\rangle$. The local $C_{3}$ symmetry axes at each site stipulate relations between the NN averages corresponding to the irreducible representations of this group: one-dimensional $a_{1}$ and two-dimensional $e$ (the former dubbed as (extended) $s$ - and the latter $d$-phase in physical context although both refer to the states of $\pi$ electrons residing in the graphene $p_{z}$ orbitals) the latter represented by two components: $x^{2}-y^{2}$ - and $x y$-waves. In the case of the $s$ phase, all $\Delta_{\mathrm{AB}}\left(\delta_{i}\right)$ are equal, while in the $x^{2}-y^{2}$ case: $\Delta_{\mathrm{AB}}\left(\delta_{1}\right)=0$ and $\Delta_{\mathrm{AB}}\left(\delta_{2}\right)=-\Delta_{\mathrm{AB}}\left(\delta_{3}\right)$; finally in the $x y$ case: $\Delta_{\mathrm{AB}}\left(\delta_{1}\right)=-2 \Delta_{\mathrm{AB}}\left(\delta_{2}\right)=-2 \Delta_{\mathrm{AB}}\left(\delta_{3}\right)$.

The model Hamiltonian used for testing the $\Theta \Phi$ capacity to reproduce the superconducting phases of graphene $\pi$-electrons was the $t$ - $J$ model Hamiltonian, featuring the one-electron hopping between NN sites of the honeycomb lattice as well as a sizable exchange interaction $J$ coming from on-site Hubbard repulsion $U: J=\frac{2 t^{2}}{U} .{ }^{40}$ The value of $t$ in graphene is known for a long time: $t=2.4 \mathrm{eV},{ }^{43,44}$ and reference therein, while the estimated $U=3.3 t .{ }^{30,40}$

Within the mean-field approach in $\Theta \Phi$ according to the formalism outlined in the Theory Section we could achieve perfect agreement with the reference data, ${ }^{30}$ as can be seen from the right panel of Fig. 5, where we compare the critical superconducting temperature $T_{c}$ as a function of doping $\eta$ (per $\mathrm{C}$ atom) at $J=1$ in the scale ${ }^{30}$ precisely corresponding to the $\Theta \Phi$ value of $J=\frac{2}{3}$ (for more detailed explanation see ${ }^{15}$ ). This agreement holds for both $s$ - and $x^{2}-y^{2}$-wave phases (we could not find literature data for $T_{c}$ in the $x y$-wave phase). We would like to emphasize that each point in the right panel of Fig. 5 is a result of a calculation similar to that represented on the left panel of Fig. 3 with many points performed to identify the critical temperature for a given level of doping. This proves the solidity of our benchmark.

To have an insight into the electronic structure of these two solutions, we compare the density of states (DOS) and the band structure in the extended-s and $x^{2}-y^{2}$-wave superconFigure 6 - here ducting phases, as shown in Fig.6. A characteristic feature of the broken symmetry states is the opening of the gap in the spectrum of (quasi)-particle energies. We observed this in the cases of Hubbard chain and square lattice. Similarly, in the case of graphene the degeneracy of the upper and lower electronic bands in the Dirac points of the Brillouin zone ( $K$ points) characteristic for graphene is lifted. One can see that in the $s$-wave superconducting phase, 
there is a full gap in the DOS, while in the $x^{2}-y^{2}$-wave superconducting phase the DOS goes to zero linearly in the vicinity of zero energy. Moreover, for the $s$-wave phase the true gap opens at $K\left(\right.$ and $K^{\prime}$ ) points in the Brillouin zone, while for the $x^{2}-y^{2}$-wave case two nodal points appear on the line $M \rightarrow K \rightarrow \Gamma^{\prime}$ remarkably shifted from the $K$ (and $K^{\prime}$ ) points - that is no true gap opens rather a DOS depletion whose exact position depends on doping $\eta$ and $J$. Notice that in the latter case, a difference appears between $K$ and $K^{\prime}$ as well as between $M=\pi\left(\frac{1}{2}, \frac{1}{2}\right)$ and $M^{\prime}=\pi\left(\frac{1}{2}, 0\right)$. Energetically, the extended- $s$ phase is the lowest one at $J>0.75 t$ (in the notations of Ref. ${ }^{40}$ ), while for $J<0.75 t$ the $d$-wave phases (almost indistinguishable between each other in free energy) are most favorable.

\section{Magnetic phases of iron}

After testing $\Theta \Phi$ against toy models like cyclic or two-dimensional Hubbard or $t$ - $J$ graphene ones we switched to true multiband models exemplified by metallic iron. The magnetism of iron is of vital fundamental and technological importance and was extensively studied (see e.g. review $^{45}$ and references therein as well as $^{46,47}$ ). Iron features versatile magnetic/structure phase diagram of which we have chosen $\alpha$ - and $\gamma$-Fe to benchmark $\Theta \Phi$. In $\alpha$-Fe we compared the FM phase as obtained in $\Theta \Phi$ and $\mathrm{DFT}+U$ calculations in vASP, while in $\gamma$-Fe after comparing the NM and FM phase with VASP DFT $+U$, with use of $\Theta \Phi$ we addressed the AFM phase as well.

The DFT simulations werer performed by us with use of $\mathrm{VASP}^{48}$ in the PBE/GGA 49 version (for details see ${ }^{15}$ ). From them we extracted the effective tight-binding Hamiltonian used as input for $\Theta \Phi$ with use of WANNIER90. ${ }^{31}$

For the sake of comparability with VASP results we used isotropic $U_{j j^{\prime}}=U$ and $J_{j j^{\prime}}=J$, although $\Theta \Phi$ in principle can handle arbitrary interaction matrices.

For $\alpha$-Fe we used the bcc lattice with $a=2.858 \AA$ and a $8 \times 8 \times 8$ Monkhorst-Pack $k$-point mesh ${ }^{50}$ (both in $\Theta \Phi$ and VASP). In this case we considered a ferromagnetic solution (FM) and a non-magnetic solution (NM) and used the energy of the latter as a reference point for comparing the relative stability of the former. It can be seen from Table 1 that 
the agreement between $\Theta \Phi$ and VASP is in general good: ${ }^{1}$ the FM state gains the energy with respect to the $\mathrm{NM}$ one and the $d$-shell magnetic moment is very close. The energy gain in $\Theta \Phi$ is approximately $20 \%$ greater, due to more adequate of the intra-atomic orbital hopping terms as explained in more details. ${ }^{15}$ The comparison of the band structures is presented in Figure 7. The overall agreement is very good. The minor discrepancy can be attributed to the different local basis sets used in VASP and in $\Theta \Phi$. In VASP, the DFT $+U$ is formulated for the charges inside PAW spheres, while in $\Theta \Phi$ for the charges occupying the Maximally Localized Wannier Orbitals (MLWO). This yields a somewhat smaller splitting between lower and upper Hubbard bands in $\Theta \Phi$. In addition, at larger $U$, the margins of the disentanglement window, where the WANNIER90 bands start to deviate from the VASP ones, may come closer to the Fermi level, thus inducing further bias.

For $\gamma$-Fe we considered the fcc lattice with $a=3.583 \AA$ and a $8 \times 8 \times 8$ Monkhorst-Pack $k$-point mesh. ${ }^{50}$ In addition to the FM and NM states mentioned above, in this case we also considered an antiferromagnetic state $(\mathrm{AFM})$ with the pitch vector $\mathbf{Q}=\pi(0,1,1)$ in terms of the vectors of the reciprocal lattice given relative to the primitive cell: the state with an alternation of oppositely magnetized ferromagnetic planes along the $z$-axis of the conventional cubic unit cell.

We found that in $\gamma$-Fe, the NM state has the highest energy among all the states considered. It can be seen from Table 2 that, at $U=6 \mathrm{eV}$ and $J=1 \mathrm{eV}$, the FM and AFM spiral states with the above value of $\mathbf{Q}$ are lower than the $\mathrm{NM}$ one and are close in energy, being the FM state slightly lower (by $0.04 \mathrm{eV}$ in $\Theta \Phi$ and $0.08 \mathrm{eV}$ in VASP respectively). We have checked that by increasing $U$, the AFM state eventually wins over the FM one, although the band structure agreement becomes somewhat worse because the edges of the WANNIER90 disentanglement window are pushed closer to the Fermi level. We point out that both the FM and AFM states energy gains coming from vASP are well reproduced in $\Theta \Phi$. Finally, the $d$-orbital magnetic moment in $\Theta \Phi$ appears to be identical to the vASP one up to the second digit. The very good correspondence of the band structure of FM $\gamma$-Fe modeled by two methods is presented in Figure 8.

Table 2 here

\footnotetext{
${ }^{1}$ The values of the magnetic moment in this and subsequent Tables serve only to show the closeness between the results stemming from vASP and $\Theta \Phi$ calculations.
}

Figure 8 - here 


\section{Spin-liquid phases of CuNCN}

The most important feature added to $\Theta \Phi$ at the present stage is the capacity to have the spin liquid states/phases, alias resonating valence bond (RVB) states, as solutions of the electronic problem: the capacity so far not accessible to any of the publicly available codes. The existence of such phases is conjectured for high- $T_{c}$ cuprate superconductors, ${ }^{10,51}$ herbertsmithite family of compounds ${ }^{9}$ to mention a few. Among them we recollect an enigmatic copper compound a nitrogen-containing analog of copper oxide synthesized recently. ${ }^{52}$ It can be derived from an oxide of the rock salt structure by replacing the formal $\mathrm{O}^{2-}$ anions by the carbodiimide dianions $\mathrm{NCN}^{2-}$ which together with the Jahn-Teller distortion characteristic for hexacoordinated $\mathrm{Cu}^{2+}$ produces relatively simple layered structure represented in Figure 9 (left). The physics of this material is rather peculiar: ${ }^{53}$ being an insulator, it shows temperature independent (Pauli-like) paramagnetism from the room temperature down to approximately $100 \mathrm{~K}$ when the latter switches to the gapped (Arrhenius-like) regime, although no magnetically ordered state could be detected down to the lowest investigated temperature. At the same time, some characteristic structural effects could be detected along with the variations of the magnetic properties. The entirety of the experimental findings in relation to $\mathrm{CuNCN}$ with a high probability allows for a consistent interpretation of its physics as a sequence of transitions between the quasi-one-dimensional and two-dimensional spin-liquid

phases. For the purpose of the present paper we show that the $\Theta \Phi$ package reproduced these features consistently and numerically.

The physical properties of CuNCN fairly map onto the $c$ - $a$-ca Heisenberg model on a rectangular lattice ${ }^{54}$ in the $a c$ crystallographic plane with the exchange interactions between the nearest neighbors: $J_{c}$ along the $c$-axis, $J_{a}$ along the $a$-axis (each $\mathrm{Cu}$ atom has two neighbors along each of the two directions), and $J_{a c}$ along the diagonals of the rectangles whose vertices are occupied by the $\mathrm{Cu}$ atoms (each atom has four neighbors along the $a c$ diagonals which otherwise would be considered as next nearest neighbors whose presence leads to frustration considered to be a prerequisite for the formation of a spin-liquid phase ${ }^{8}$ ). The scheme of the relevant Heisenberg model is shown in Figure 9 (right).

The spin-liquid phases are considered to be a physical manifestation of the RVB solutions 
of electronic problems for respective materials (Hamiltonians). The electronic states of the RVB type are superpositions of the individual configurations where all the electron spins residing in all nodes of a lattice form singlet pairs: ${ }^{55}$

$$
\left(c_{r \uparrow}^{\dagger} c_{r^{\prime} \downarrow}^{\dagger}-c_{r \downarrow}^{\dagger} c_{r^{\prime} \uparrow}^{\dagger}\right)
$$

The most known example of such a state is that of benzene depicted in Figure 10. The corresponding pairings are shown in Figure 10 by colored ellipsoids which indicate formation of spin-pairings between the (electrons residing in the orbitals attached to the) vertices, they join. Contrary to the widespread belief, the RVB state of benzene has very little to do with the Hückel state, that is, the Slater determinant of the occupied molecular (crystal) orbitals or the symmetry adapted linear combinations of the orbitals attached to the vertices. This is the only option available in the standard solid state quantum chemistry codes. As explained in Refs. ${ }^{55,56}$ the weight of the RVB state in the single determinant wave function (for benzene) is not more than $12.5 \%$ and even more - the corresponding amount - the contribution of the RVB states to it vanishes when it comes to an infinite system, a crystal. ${ }^{56}$ Thus, in order to be able to access either the single determinant (ultimately, the only available in the standard codes) and an RVB solution within a single procedure special precautions need to be made. It can be shown, ${ }^{57}$ that the RVB state can be covered by an independent (quasi)particle density Eq. (11) if off-diagonal anomalous averages are considered together with their normal off-diagonal density counterparts. This manifests in appearance of nonvanishing order parameters $\zeta_{\tau}=\sqrt{\xi_{\tau}^{2}+\left|\Delta_{\tau}\right|^{2}} ; \xi_{\tau}=\left\langle c_{r s}^{\dagger} c_{r+\tau s}\right\rangle$ combining the normal averages $\xi_{\tau}$ and the anomalous ones $\Delta_{\tau}$ - the characteristic of a spin-liquid phase. ${ }^{58}$ When it comes to $\mathrm{CuNCN}$ as described by the $c-a-c a$ model (Figure 9 - right) the RVB states can be expected (Figure 11). In each of the RVB states we show pairings related to the respective exchange interactions which are indicated by the colors of ellipses. In the respective states characterized by the presence of non-vanishing order parameters $\zeta_{\tau}$ for the allowed values of the neighbor vectors $\tau$ all indicated pairings are present as indicated by the $\oplus$ signs. The analysis ${ }^{54}$ of the $c-a-c a$ model in the high temperature approximation reveals a rich diagram of the RVB/spin-liquids phases. Specifically, depending on the relation between the exchange constants $J_{c}, J_{a}$, and $J_{a c}$ and temperature one can expect formation of eight 
electronic phases in which the corresponding anomalous averages along respective directions in the crystal are non-vanishing in various combinations as depicted in Figure 11.

The $\Theta \Phi$ package as applied to this model exactly reproduces the critical temperatures at which the respective anomalous averages/order parameters bounce from zero, coming from the high temperature expansion of the free energy. Important acquisitions for the lowest temperature region $(T<0.02 J)$ available only with help of $\Theta \Phi$ as compared to the hightemperature estimates are (i) the temperature dependence of the order parameters $\zeta_{\tau}$ in the vicinity of zero temperature, following the same law Eq. (15) as the anomalous averages in the Hubbard chain model with on-site attraction and (ii) the parametric phase diagram at the temperature flowing to zero. As for the latter, the numeric study with $\Theta \Phi$ only partially confirms the result of our previous study. ${ }^{59}$ Indeed, as one can conjecture from inspection of the sequence of phase diagrams in Figure 12 (right) with decrease of the temperature the richness of the spin-liquid phases degenerates, so that all phases except two 2D spin-liquids depicted yellow (three order parameters are non-vanishing) and orange (two non-vanishing Figure 12 - here order parameters $\zeta_{a}$ and $\zeta_{c}$ ) are squeezed out to the respective lines where either $J_{a}$ or $J_{c}$ vanish. The stability analysis of the "orange" spin-liquid with respect to emergence of a non-vanishing $\zeta_{a c}$ the "yellow" spin-liquid ${ }^{59}$ shows the coefficient at $\zeta_{a c}^{2}$ in the free energy expansion performed in the "orange" phase to be always positive. This phase is stable with respect to the transition into the "yellow" phase. This had been interpreted ${ }^{59}$ as an indication of the global "orange" spin-liquid ground state of the $c-a-c a$ model. The numerical results of the present work, however, show that the stability of the "orange" phase was interpreted too widely. The stability of the "orange" phase ${ }^{59}$ means only that the transition to the "yellow" one cannot be of the second order, but may fairly be a first-order transition implying some non-homogeneity of the material previously suspected from experiment. ${ }^{53}$ We return to this possibility below. Thus, the zero temperature parametric phase diagram of spin-liquids contains two regions occupied, respectively, by the "yellow" and "orange" spin-liquids, the finding established numerically with help of $\Theta \Phi$. 


\section{Spiral magnetic states in CuNCN}

The spin-liquid (RVB) phases are not the only possible ones in the $c$ - $a$-ca model of $\mathrm{CuNCN}$. More traditional, spin spiral states can be easily simulated within $\Theta \Phi$, too. These states can be stabilized by building the periodic spin patterns with the pitch vector $\mathbf{Q}$. For the above mentioned "experimental" parameter values showing the temperature dependence of the spinliquid order parameters depicted in Figure 12 and fitting the experimental data for CuNCN at intermediate temperatures we, with use of $\Theta \Phi$, performed a scan through the $\mathbf{Q}$ vector in the first Brillouin zone and plotted the energy of a magnetic state of the $c$ - $a$-ca model as a function of $\mathbf{Q}$ (Figure 13). The energy landscape presents two minima at $(\pi, 0)$ and $(0, \pi)$, and one saddle point around $(\pi / 2, \pi / 2)$ together with two fairly flat regions around $(0,0)$ and $(\pi, \pi)$. The latter correspond to an absence of any magnetic solution at the corresponding values of the $\mathbf{Q}$-vector. The minimum at $(\pi, 0)$ corresponds to a spin density wave with spins arranged parallel and alternating "up"-"down" orientation at nearest neighbor distance along $a$-axis, the one at $(0, \pi)$ alternate along $c$-axis. Finally the energetically unstable saddle point corresponds to an incommensurate spin-density wave with the spin quantization axis making a complete rotation at approximately every two steps along the $c a$ direction. The minima depths are: -0.3187 at $(\pi, 0)$ and -0.4601 at $(0, \pi)$, respectively (in units of $J$ ). The depth of the saddle point is $-0.06 \mathrm{~J}$. The minimum at $(0, \pi)$ is lower because in the present example $J_{c}>J_{a}$. Therefore, the globally stable spin spiral state in CuNCN at the given values of $J$ parameters is realized at $\mathbf{Q}=(0, \pi)$ according to $\Theta \Phi$ calculation with the minimum energy value of $-0.4601 J$. Remarkably, the spin-liquid phase has the energy of $-0.125 \mathrm{~J}$. Interestingly, the spiral magnetic solution in the Heisenberg system like CuNCN at the experimental values of $J$ tends to stabilize commensurate spin waves $((0, \pi)$ and $(\pi, 0))$ as opposed to e.g. the 2D square lattice with doping as described by the Hubbard Hamiltomian considered above. This is a consequence of several factors: i) comparable values of all three antiferromagnetic $J$ values; ii) inter-site connectivity of the Heisenberg couplings which tends to favor the (commensurate) order at the corresponding distances; and iii) absence of the kinetic energy term loosing from the spiral order and the on-site Coulomb repulsion gaining from the spiral order, which creates an interplay leading to the incommensurate spin spirals. 
These issues will be considered elsewhere.

Thus, according to numerical analysis of the present paper the ground state (lowest temperature phase) of the $c$ - $a$-ca model of $\mathrm{CuNCN}$ is a state with frozen momenta, as established by the $\mu$ SR measurements. ${ }^{60}$ Due to significant difference between the spin-ordered and spinliquid states (completely different set of non-vanishing order parameters) one can conjecture that the respective solutions represent different local minima of the free energy so that the transition between them is a first order one. As a consequence, a temperature range where the phases coexist may occur; in this range, the material is heterogeneous with spatial areas occupied by either one or another (respectively, spin frozen and spin liquid ones) phase.

\section{CONCLUSIONS, AND PERSPECTIVES}

As of today, the available solid-state electronic-structure codes suffer from a limited functionality with respect to the possible magnetic superstructures. The generally implemented technology of multiplying the chemical unit cells allows to mimic only commensurate magnetic structures and even the simplest ones, since increasing the unit cell size by a factor of $n$ leads to the $n^{3}$ increase of the required computational resources due to employed diagonalization procedures. Moreover, alternative types of electronic states like BCS and/or RVB corresponding to physically interesting superconducting and spin-liquid electronic states/phases of materials are not available at all. The temperature dependence of the solutions of electronic problem (an important component of the physical behavior of superconducting, spin-liquid and magnetic materials) is not accessible either. These gaps are partially closed by the new $\Theta \Phi$ package which will be further developed.

On examples of two model Hamiltonians (the Hubbard one for one-dimensional chain and for two-dimensional square lattice and the $t$ - $J$ one for graphene) we demonstrate the capacity of $\Theta \Phi$ to reproduce the temperature dependent BCS solutions in these models. The existence of RVB (spin-liquid) states/phases in CuNCN has been confirmed as well as the existence of characteristic dependency of the RVB order parameters on temperature, known from analytic theory. At the same time, it is shown that in $\mathrm{CuNCN}$, the spin-ordered state has a lower energy for the ratio of the exchange parameters fitting the experimental data. 
Since the tight-binding Hamiltonians are the inputs for the proposed procedure, the ports to the sources of such have been implemented. With use of the tight-binding parameters as extracted from the VASP calculation of the ferromagnetic bcc and antiferromagnetic fcc iron projected on the $3 d 4 s 4 p$ local basis the band structures and the respective magnetic momenta together with their relative energies are fairly reproduced. The magnetically ordered states known from experiment are reproduced through optimization of energy with respect to the superstructure wave vector, without extending the chemical unit cell. By this the applicability of the code to the multi-band models of interacting electrons in solids is demonstrated. The $\Theta \Phi$ package is available at the the NetLaboratory portal ${ }^{61}$ for registered users.

\section{ACKNOWLEDGMENTS}

This work is partially supported by the German Research Foundation through the Collaboration Research Grant 761 (Steel ab initio) and by the Russian Science Foundation (grant № 19-73-10206). 


\section{References}

1. R. Dronskowski, Computational Chemistry of Solid State Materials (Wiley-VCH, 2005).

2. K. Schwarz and P. Blaha, in Handbook of Solid State Chemistry, edited by R. Dronskowski, S. Kikkawa, and A. Stein (Wiley-VCH, 2017), vol. 5.

3. D. Usvyat, L. Maschio, and M. Schütz, in Handbook of Solid State Chemistry, edited by R. Dronskowski, S. Kikkawa, and A. Stein (Wiley-VCH, 2017), vol. 5.

4. T. Bredow and K. Jug, in Handbook of Solid State Chemistry, edited by R. Dronskowski, S. Kikkawa, and A. Stein (Wiley-VCH, 2017), vol. 5.

5. J. Bardeen, L. Cooper, and J. Schrieffer, Physical Review 106, 162 (1957).

6. J. Bardeen, L. Cooper, and J. Schrieffer, Physical Review 108, 1175 (1957).

7. P. Fazekas and P. W. Anderson, Philosophical Magazine 30, 423 (1974).

8. L. Balents, Nature 464, 199 (2010), ISSN 0028-0836.

9. T. Imai and Y. Lee, Physics Today 69, 30 (2016).

10. P. W. Anderson, Science 235, 1196 (1987).

11. G. A. Landrum and R. Dronskowski, Angew Chem Int Ed 39, 1560 (2000).

12. R. Dronskowski, Intl. J. Quant. Chem. 96, 89 (2004).

13. A. Decker, G. A. Landrum, and R. Dronskowski, Z. Anorg. Allg. Chem. 628, 303 (2002).

14. B. P. T. Fokwa, H. Lueken, and R. Dronskowski, Chem Eur J 13, 6040 (2007).

15. E. Plekhanov, A. Tchougréeff, and R. Dronskowski, Computer Physics Communications 251, 107079 (2020).

16. E. Plekhanov and A. Tchougreéff., Comp. Mat. Sci. (Accepted).

17. G. C. Wick, Phys. Rev. 80, 268 (1950). 
18. J.-P. Blaizot and G. Ripka, Quantum Theory of Finite Systems (MIT Press, 1985).

19. Y. Nambu, Phys. Rev. 117, 648 (1960).

20. L. M. Sandratskii, physica status solidi (b) 136, 167 (1986).

21. E. Arrigoni and G. C. Strinati, Phys. Rev. B 44, 7455 (1991).

22. O. N. Mryasov, A. I. Liechtenstein, L. M. Sandratskii, and V. A. Gubanov, Journal of Physics: Condensed Matter 3, 7683 (1991).

23. N. Bogolubov, J. of Physics 11, 23 (1947).

24. N. N. Bogoliubov, J. Exptl. Theoret. Phys. (U.S.S.R.)/Soviet Physics JETP 34, 73 (1958).

25. N. N. Bogoliubov, J. Exptl. Theoret. Phys. (U.S.S.R.)/Soviet Physics JETP 34, 58 (1958).

26. V. V. Tolmachev and S. V. Tiablikov, J. Exptl. Theoret. Phys. (U.S.S.R.)/Soviet Physics JETP 34, 66 (1958).

27. P.-G. de Gennes, Superconductivity of Metals and Alloys, Advanced book classics (Advanced Book Program, Perseus Books, 1999), ISBN 9780738201016.

28. L. D. Landau and E. M. Lifshitz, Course of Theoretical Physics. Vol. 5. Statistical Physics. (Butterworth-Heinemann, 1980), 3rd ed.

29. W. H. Press, S. A. Teukolsky, W. T. Vetterling, and B. P. Flannery, Numerical Recipes in FORTRAN; The Art of Scientific Computing (Cambridge University Press, New York, NY, USA, 1993), 2nd ed., ISBN 0521437164.

30. A. M. Black-Schaffer and C. Honerkamp, Journal of Physics: Condensed Matter 26, 423201 (2014).

31. A. Mostofi, J. R. Yates, Y.-S. Lee, I. Souza, D. Vanderbilt, and D. Marzari, Comput. Phys. Commun. 178, 685 (2008). 
32. V. L. Deringer, A. L. Tchougreeff, and R. Dronskowski, Journal of Physical Chemistry A 115, 5461 (2011).

33. S. Maintz, V. L. Deringer, A. L. Tchougréeff, and R. Dronskowski, Journal of Computational Chemistry 34, 2557 (2013).

34. S. Maintz, V. L. Deringer, A. L. Tchougréeff, and R. Dronskowski, Journal of Computational Chemistry 37, 1030 (2016).

35. A. L. Tchougreeff and R. Dronskowski, Journal of Physical Chemistry A 117, 7980 (2013).

36. I. Misurkin and A. Ovchinnikov, Theor. Exp. Chem. 4, , 3. (1968).

37. R. Hoffmann, T. Hughbanks, M. Kertesz, and P. H. Bird, J. Am. Chem. Soc. 105, 4831 (1983).

38. Y. Cao, V. Fatemi, A. Demir, S. Fang, S. L. Tomarken, J. Y. Luo, J. D. SanchezYamagishi, K. Watanabe, T. Taniguchi, E. Kaxiras, et al., Nature 556, 80 (2018).

39. Y. Cao, V. Fatemi, S. Fang, K. Watanabe, T. Taniguchi, E. Kaxiras, and P. JarilloHerrero, Nature 556, 43 (2018).

40. A. M. Black-Schaffer and S. Doniach, Phys. Rev. B 75, 134512 (2007).

41. I.-D. Potirniche, J. Maciejko, R. Nandkishore, and S. L. Sondhi, Phys. Rev. B 90, 094516 (2014).

42. R. Nandkishore, L. S. Levitov, and A. V. Chubukov, Nature Physics 8, 158 (2012).

43. A. L. Tchougreeff and R. Hoffmann, Journal of Physical Chemistry 96, 8993 (1992).

44. A. L. Tchougreeff, Journal of Physical Chemistry 100, 14048 (1996).

45. W. Pepperhoff and M. Acet, Constitution and Magnetism of Iron and its Alloys, Engineering Materials and Processes (Springer, 2001), ISBN 9783662043455.

46. M. Marsman and J. Hafner, Phys. Rev. B 66, 224409 (2002). 
47. D. Hobbs, J. Hafner, and D. Spišák, Phys. Rev. B 68, 014407 (2003).

48. G. Kresse and D. Joubert, Phys. Rev. B 59, 1758 (1999).

49. J. P. Perdew, K. Burke, and M. Ernzerhof, Phys. Rev. Lett. 77, 3865 (1996).

50. J. Monkhorst and J. D. Pack, Phys. Rev. B 13, 5188 (1976).

51. Z. Zou and P. W. Anderson, Phys. Rev. B 37, 627 (1988).

52. X.-H. Liu, R. Dronskowski, R. Kremer, M. Ahrens, C.-D. Lee, and M.-H. Whangbo, J. Phys. Chem. C 112, 11013 (2008).

53. A. Zorko, P. Jeglič, A. Potočnik, D. Arčon, A. Balčytis, Z. Jagličić, X. Liu, A. L. Tchougréeff, and R. Dronskowski, Physical Review Letters 107, 047208 (2011).

54. A. L. Tchougreéff and R. Dronskowski, Journal of Physics-condensed Matter 25, 435602 (2013).

55. E. A. Plekhanov and A. L. Tchougréeff, in Handbook of Solid State Chemistry, edited by R. Dronskowski, S. Kikkawa, and A. Stein (Wiley, 2017), vol. 5, ISBN 9783527691036.

56. A. L. Tchougréeff, International Journal of Quantum Chemistry 116, 137 (2016), ISSN 0020-7608; 1097-461X.

57. M. Ogata and H. Fukuyama, Reports on Progress in Physics 71, 36501 (2008), ISSN 0034-4885.

58. A. Tchougréeff and R. Dronskowski, On the properties of the longitudinal rvb state in the anisotropic triangular lattice. mean-field rvb analytical results. (2010), URL https://arxiv.org/abs/1008.0182.

59. A. L. Tchougréeff and R. Dronskowski, Low Temperature Physics 40, 73 (2014).

60. A. Zorko, P. Jeglič, M. Pregelj, D. Arčon, H. Luetkens, A. L. Tchougréeff, and R. Dronskowski, Phys. Rev. B 97, 214432 (2018).

61. A. Tchougreeff, Netlaboratory (1998), URL https://netlab.cartesius .info/doxygen/thetaphi/in 
62. K. Momma and F. Izumi, J Appl Crystallogr 44, 1272 (2011). 
Figure 1: $\Theta \Phi$ program flowchart diagram. Basically, it goes about the same entities (enclosed in rectangles), operations (enclosed in ellipses), checks (enclosed in a rhombus) and transitions shown by arrows, as appear in all solid-state quantum-chemistry packages. They all are modified within $\Theta \Phi$ in order to accommodate temperature dependence, superconducting and spin-liquid states as well as magnetic superstructure as explained in the subsequent Subsections.

Figure 2: The structure of a hypothetized ${ }^{37}$ metallic carbon allotrope featuring the $\mathrm{sp}^{2}$ hybridization of each carbon atom forming quasi-isolated chains of the $\pi$-AOs further characterized by the nearest neighbor hopping parameter $t$ and the on-site electron-electron interaction parameter $U$. Picture is produced with use of the VESTA 3 software. ${ }^{62}$

Figure 3: Left: temperature at $U=-1$ and right $U$ dependence at $\theta=10^{-5}$ of the superconducting paring $\Delta$ for local superconducting phase of 1D Hubbard model. The calculation is performed for $n=1$ (half-filling) of 50000 sites. The inset shows the details of the $(\Delta)$ approach to zero while $U \rightarrow 0$. The blue lines on the left and the inset are the respective analytic formulae Eqs. (15) and (16). Adapted from. ${ }^{15}$

Figure 4: Left: A benchmark of $\Theta \Phi$ incommensurate spin state phase diagram. The axes represent inverse interaction $1 / U$, doping $\eta$ and pitch vector offset from $(\pi, \pi)$ normalized to $\pi$. Right: Spin rotation pattern in a $20 \times 202 \mathrm{D}$ square lattice chunk with a pitch vector of $\mathbf{Q}=(\pi, \pi)+(\Delta Q, \Delta Q)$ with $\Delta Q=\frac{\pi}{10}$, that is for $\eta \approx 0.02$ and $U \approx 10$. Looking attentively one can notice that the NN spins are approximately antiparallel and become exactly antiparralel after ten steps for either combination of unit cell vectors. For twenty such steps spins become parallel suggesting the the magnetic supercell required for reproducing such a spiral state to be $20 \times 20$ as well. We stress once again, that within $\Theta \Phi$ this result is achieved by diagonalizing Fockian matrices for one unit cell containing one $p_{\mathrm{z}} \mathrm{AO}$. Adapted from. ${ }^{16}$ 
Figure 5: Left panel: graphene unit cell showing two types of sites (A and B), vectors $\left\{\delta_{i}\right\}$ connecting NN sites, and the unit cell vectors $\left\{a_{1(2)}\right\}$. The dotted rhombus shows a "chemical" unit cell as comprising two atoms of A and B sublattices, respectively. Right panel: Comparison of the doping dependence $\theta_{c}(\eta)$ for $s$-wave and $x^{2}-y^{2}$-wave phases of graphene with the data. ${ }^{30}$ The points were extracted from the graphs ${ }^{30}$, while the lines are calculated by $\Theta \Phi$. For the details of the calculations and the difference in the exchange parameter definitions see the text. Adapted from. ${ }^{15}$

Figure 6: Left panel: band structure along the path $\Gamma \rightarrow M \rightarrow K \rightarrow \Gamma^{\prime} \rightarrow M^{\prime} \rightarrow \Gamma$ and DOS for the $s$-wave superconducting phase of graphene. Right panel: Band structure along the path $\Gamma \rightarrow M \rightarrow K \rightarrow \Gamma^{\prime} \rightarrow M^{\prime} \rightarrow \Gamma$ and DOS for $x^{2}-y^{2}$-wave superconducting phase of graphene. Notice that in this case, a difference appears between $K=\pi\left(\frac{1}{3}, \frac{2}{3}\right)$ and $K^{\prime}=\pi\left(\frac{2}{3}, \frac{1}{3}\right)$ as well as between $M=\pi\left(\frac{1}{2}, \frac{1}{2}\right)$ and $M^{\prime}=\pi\left(\frac{1}{2}, 0\right)$. In either case $\Gamma^{\prime}$ point is the center of the adjacent BZ with the $k$-space coordinates $(1,0) ; \eta=0.1 /$ atom, $\theta=10^{-5}$. In either case it goes about the states of $\pi$-electrons of a graphene sheet. Adapted from. ${ }^{15}$

Figure 7: Band structure comparison between $\Theta \Phi$ (left panel) and VASP (right panel) for ferromagnetic $\alpha$-Fe. Adapted from. ${ }^{15}$

Figure 8: Band structure comparison between $\Theta \Phi$ (left panel) and vASP (right panel) for antiferromagnetic calculations in $\gamma$-Fe. Adapted from. ${ }^{15}$

Figure 9: Structure of CuNCN material (left) and the respective antiferromagnetic interactions within an $a c$-layer according to $c$ - $a$ - $c a$ model (right). Adapted from. ${ }^{15}$

Figure 10: Simplest and most widespread example of an RVB state in a molecule: benzene with antiferromagnetic coupling between adjacent sites bearing one electron (spin) each. Its wave function is a superposition (as shown by $\oplus$ symbols) of five linearly independent pairings known as Rumer basis. 
Figure 11: Exemplary RVB states of the $c-a-c a$ model of CuNCN. Three order parameters $\zeta_{c}, \zeta_{a}, \zeta_{c a}$ are possible. They may be either vanishing or non-vanishing producing altogether eight possible combinations of which we represent five nontrivial ones. One more with all three $\zeta_{\tau}$ 's vanishing is a high-temperature disordered phase (shown in gray in the parametric phase diagrams in Figure 12) and two more (1D and Q1D with spin-pairings along the different crystallographic directions).

Figure 12: Various CuNCN properties calculated by $\Theta \Phi$. Left: spin-liquid order parameters $\zeta_{a}, \zeta_{c}$ and $\zeta_{a c}$ as functions of temperature for $J_{a}=0.2346, J_{c}=0.376, J_{a c}=0.3894$ corresponding to the values of exchange parameters fitting an entirety of experimental data. ${ }^{54}$ Right: parametric phase diagrams for the $c$ - $a$ - $c a$ model for different temperatures.

Figure 13: Energy as a function of pitch vector in CuNCN at $J_{a}=0.2346, J_{c}=0.376, J_{a c}=$ 0.3894. Energy is expressed in units of $J \equiv J_{a}+J_{c}+J_{a c} . J$ in CuNCN is estimated to be $3270 \mathrm{~K} .{ }^{54}$ Contour lines are added for better visualization. Calculations are performed at $\theta=10^{-6} J$. The red dot on the energy axis represents the RVB state solution. 


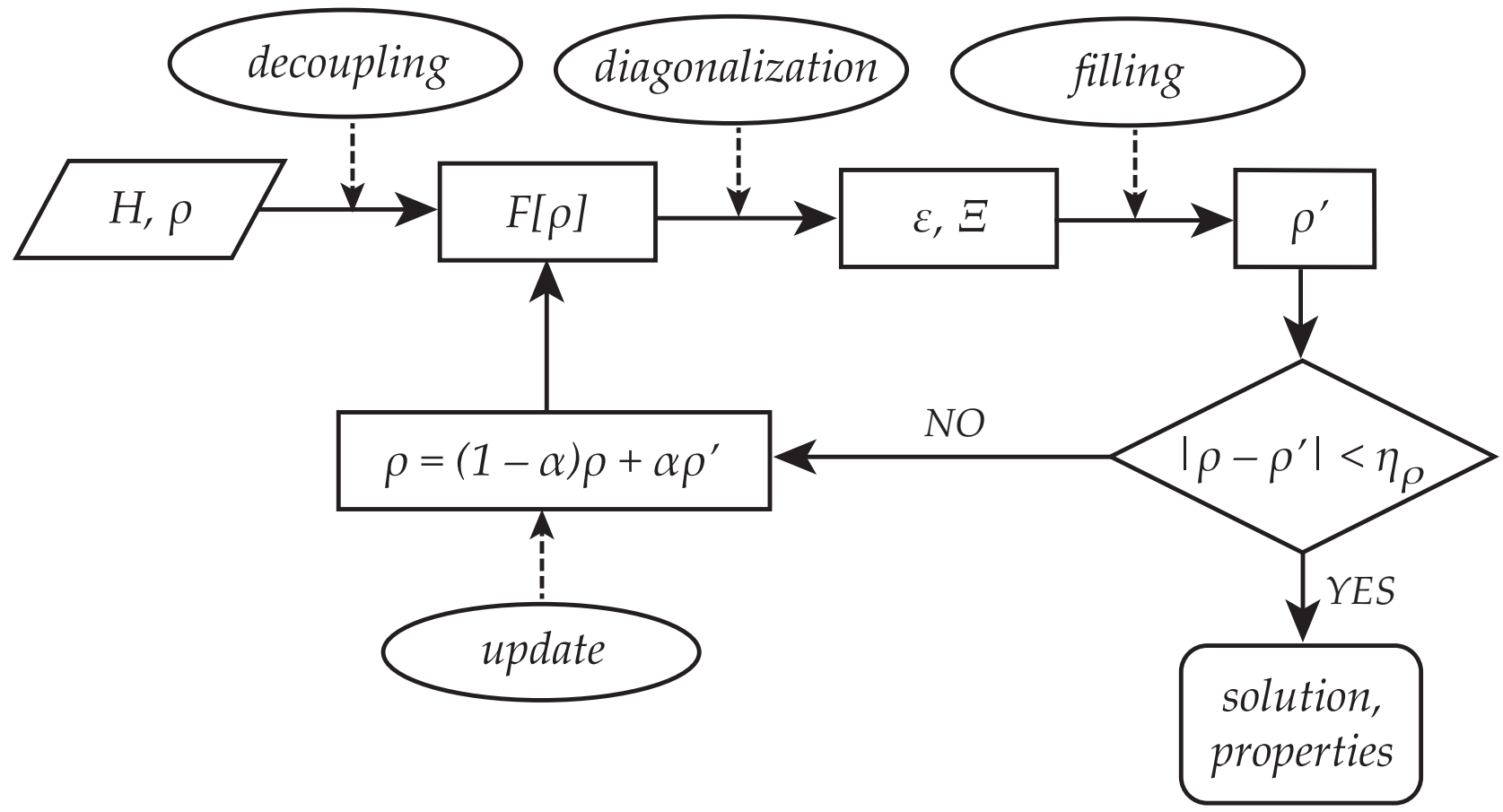

Figure 1

A. Tchougréeff, E. Plekhanov,

R. Dronskowski

J. Comput. Chem. 


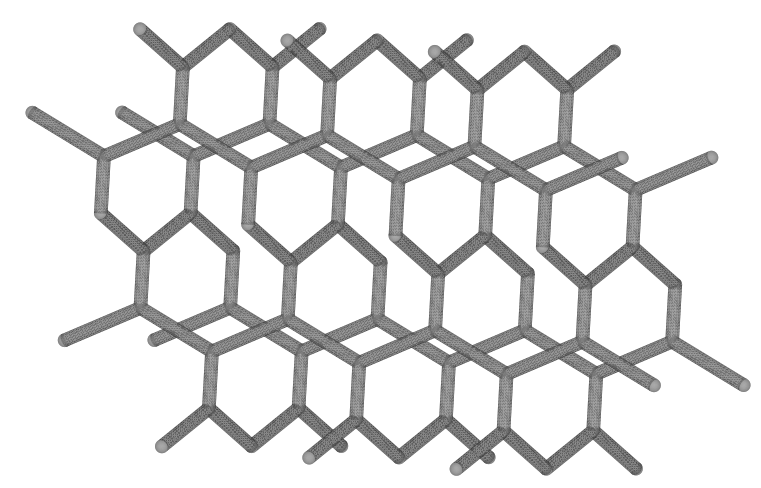

Figure 2

A. Tchougréeff, E. Plekhanov, R. Dronskowski

J. Comput. Chem. 

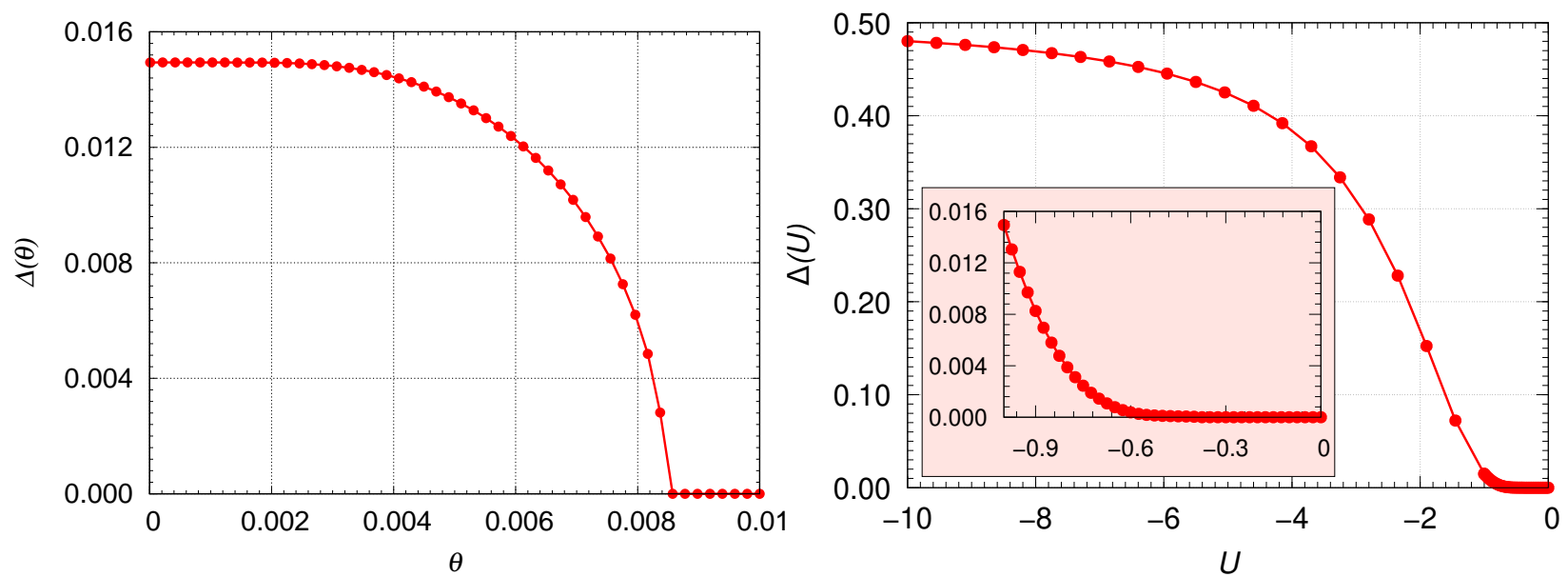

Figure 3

A. Tchougréeff, E. Plekhanov, R. Dronskowski

J. Comput. Chem. 

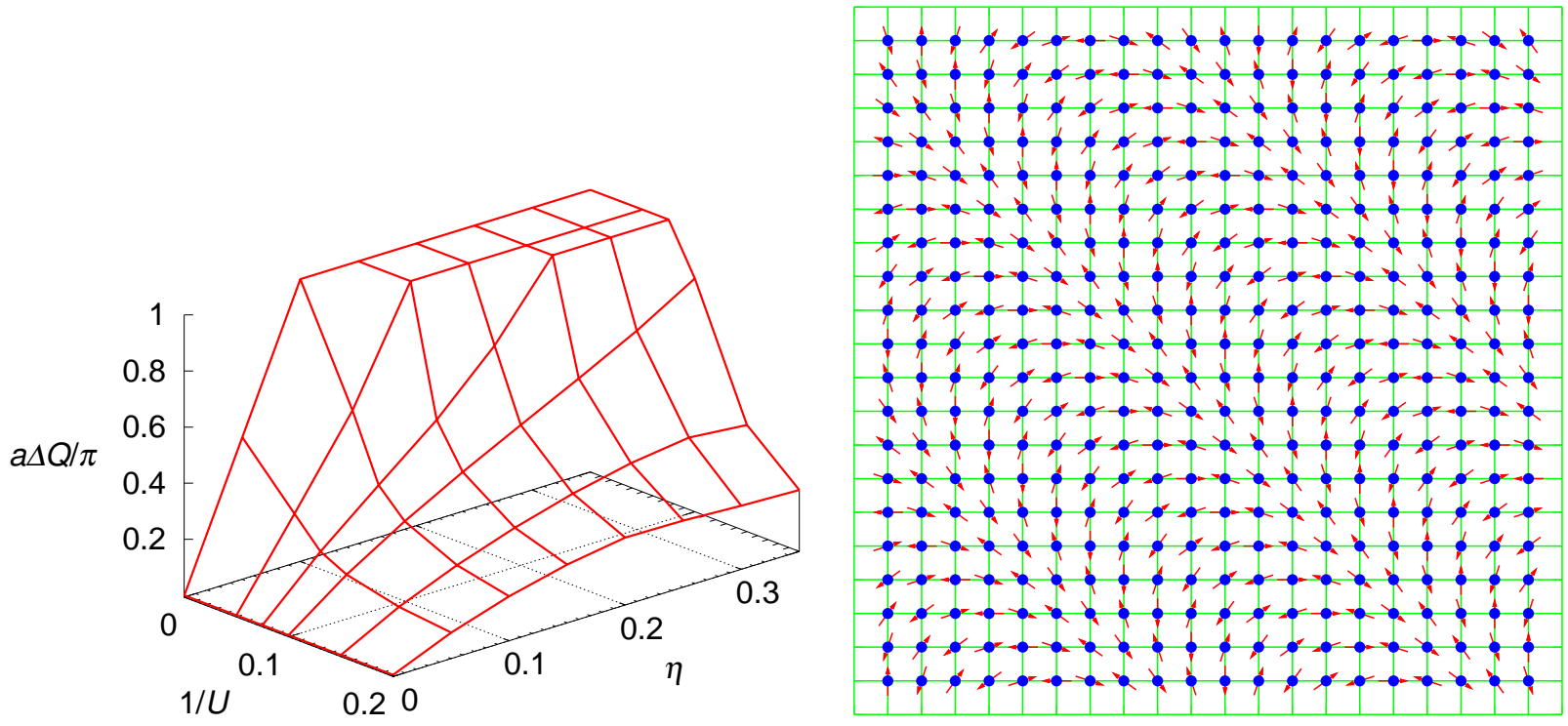

Figure 4

A. Tchougréeff, E. Plekhanov,

R. Dronskowski

J. Comput. Chem. 

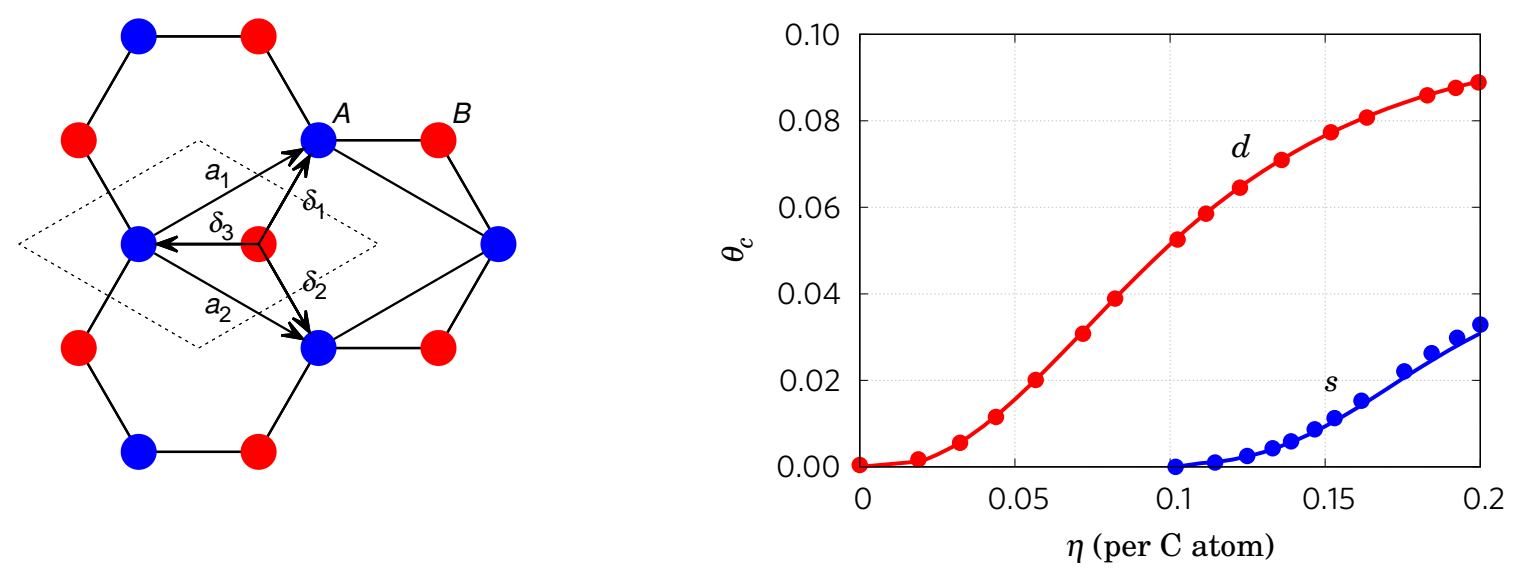

Figure 5

A. Tchougréeff, E. Plekhanov,

R. Dronskowski

J. Comput. Chem. 

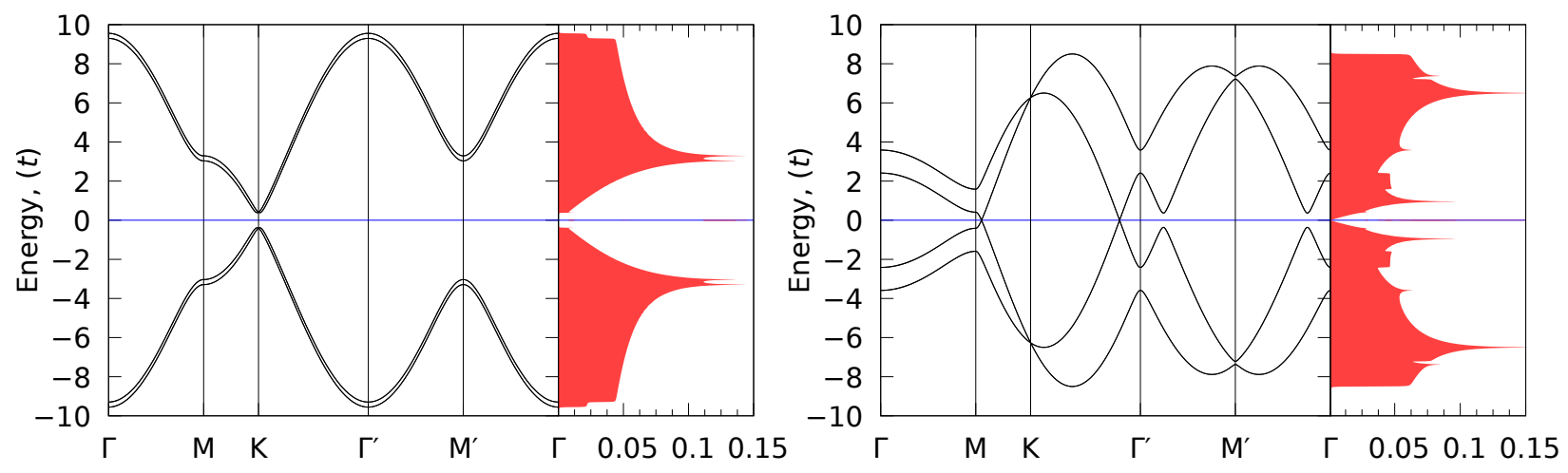

Figure 6

A. Tchougréeff, E. Plekhanov,

R. Dronskowski

J. Comput. Chem. 

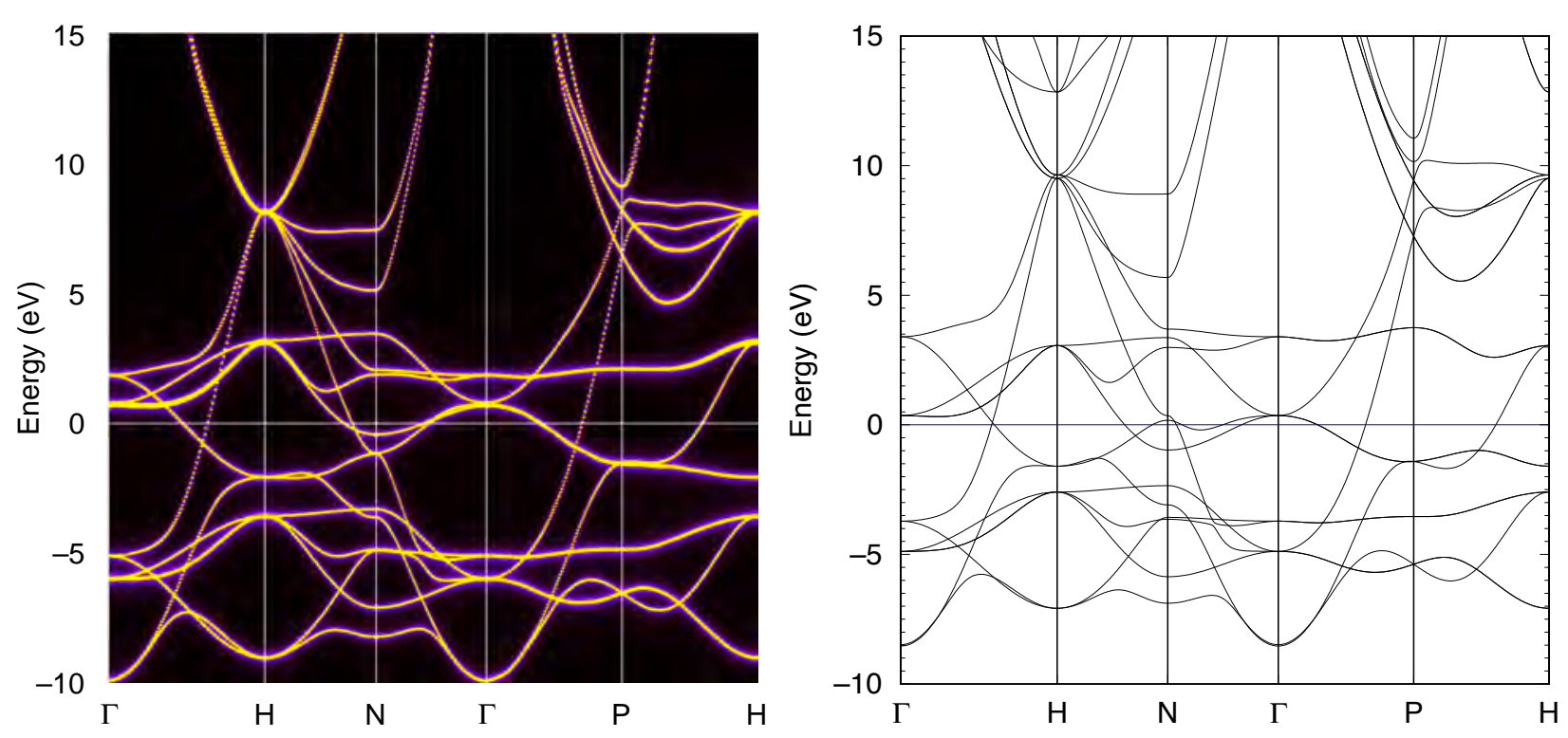

Figure 7

A. Tchougréeff, E. Plekhanov,

R. Dronskowski

J. Comput. Chem. 

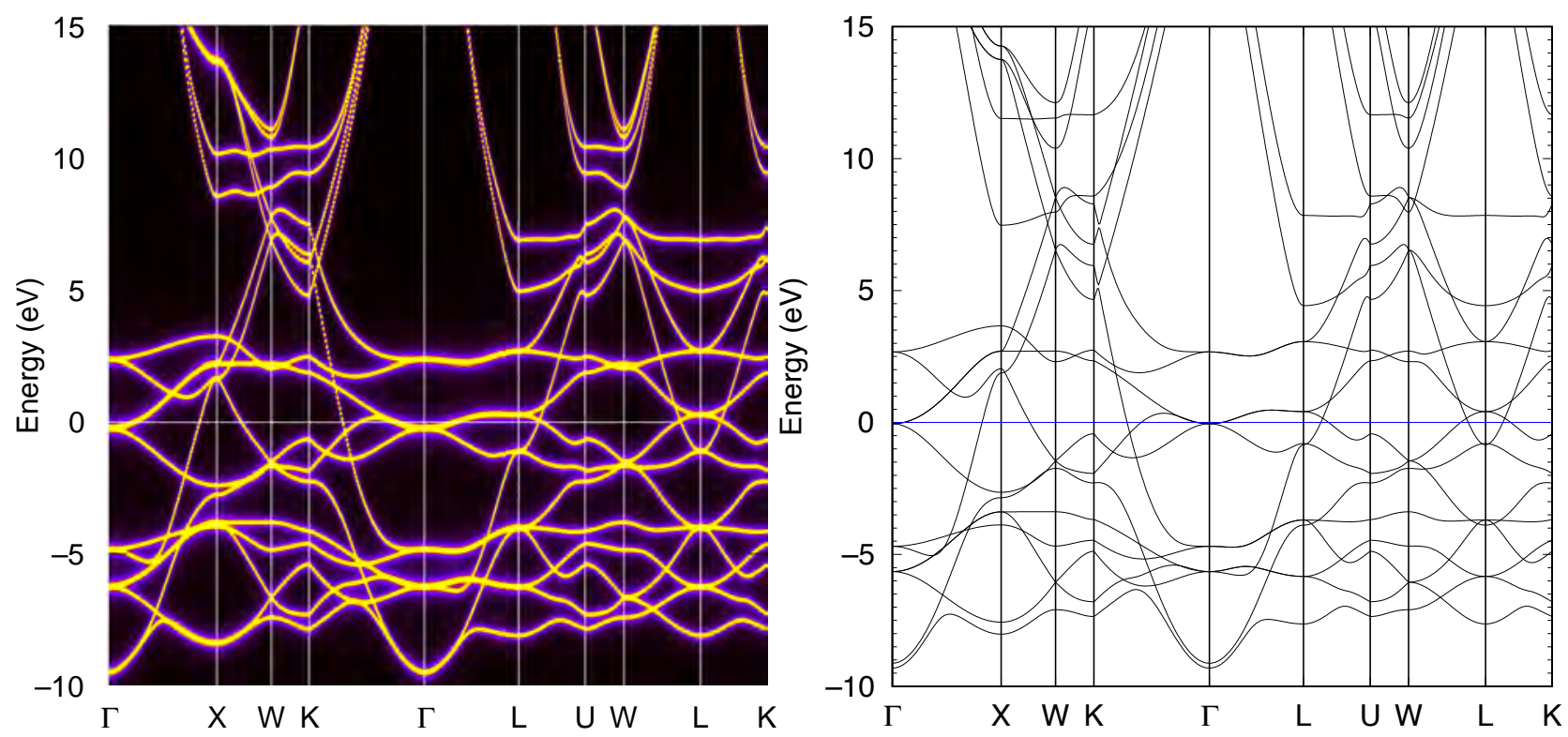

Figure 8

A. Tchougréeff, E. Plekhanov,

R. Dronskowski

J. Comput. Chem. 

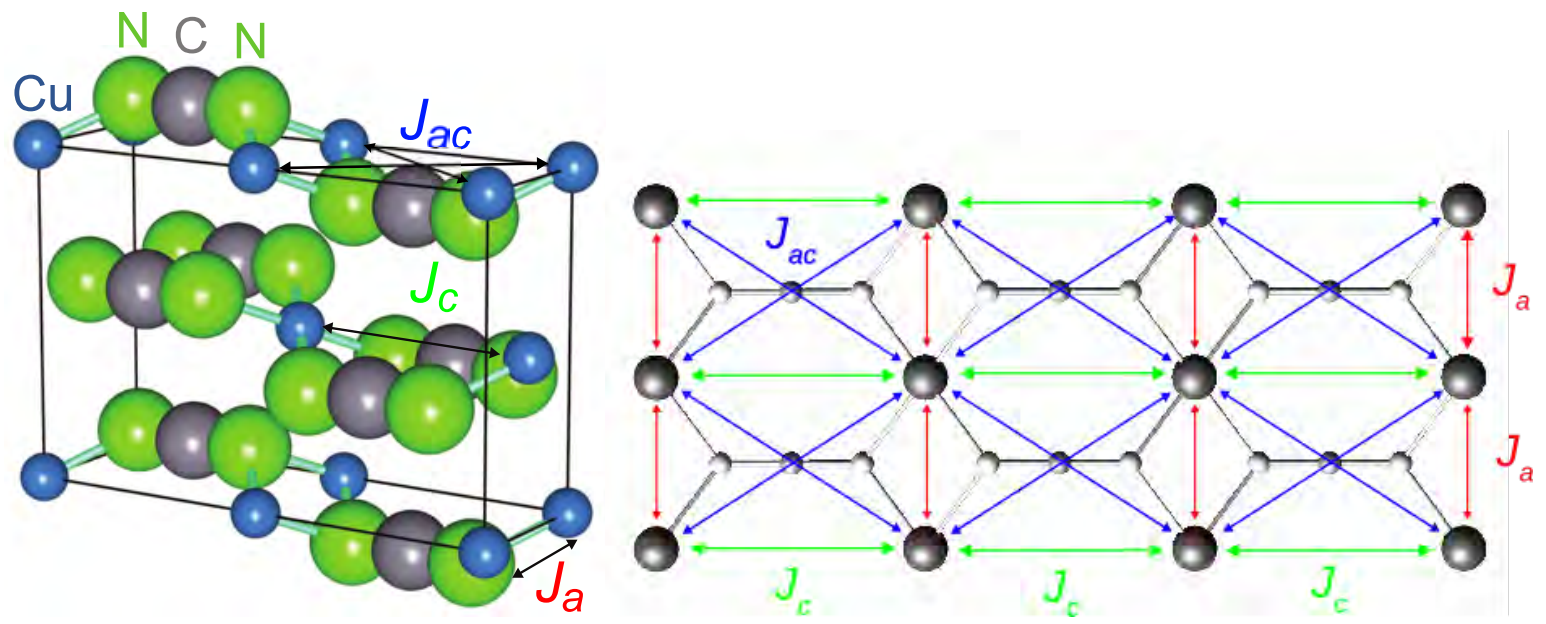

Figure 9

A. Tchougréeff, E. Plekhanov,

R. Dronskowski

J. Comput. Chem. 

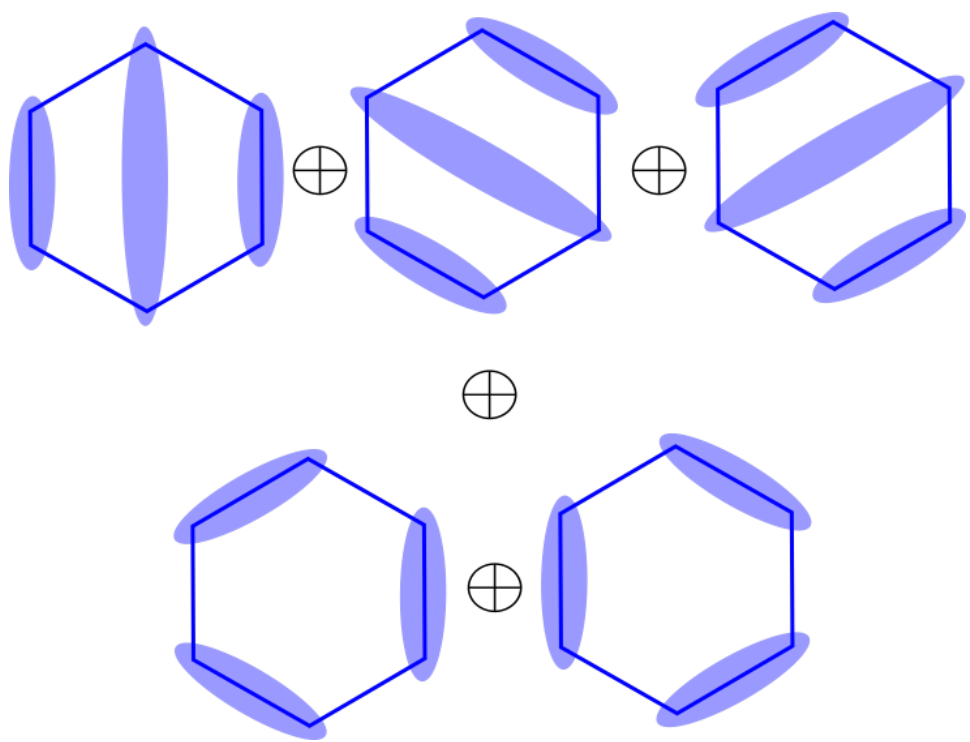

Figure 10

A. Tchougréeff, E. Plekhanov, R. Dronskowski

J. Comput. Chem. 


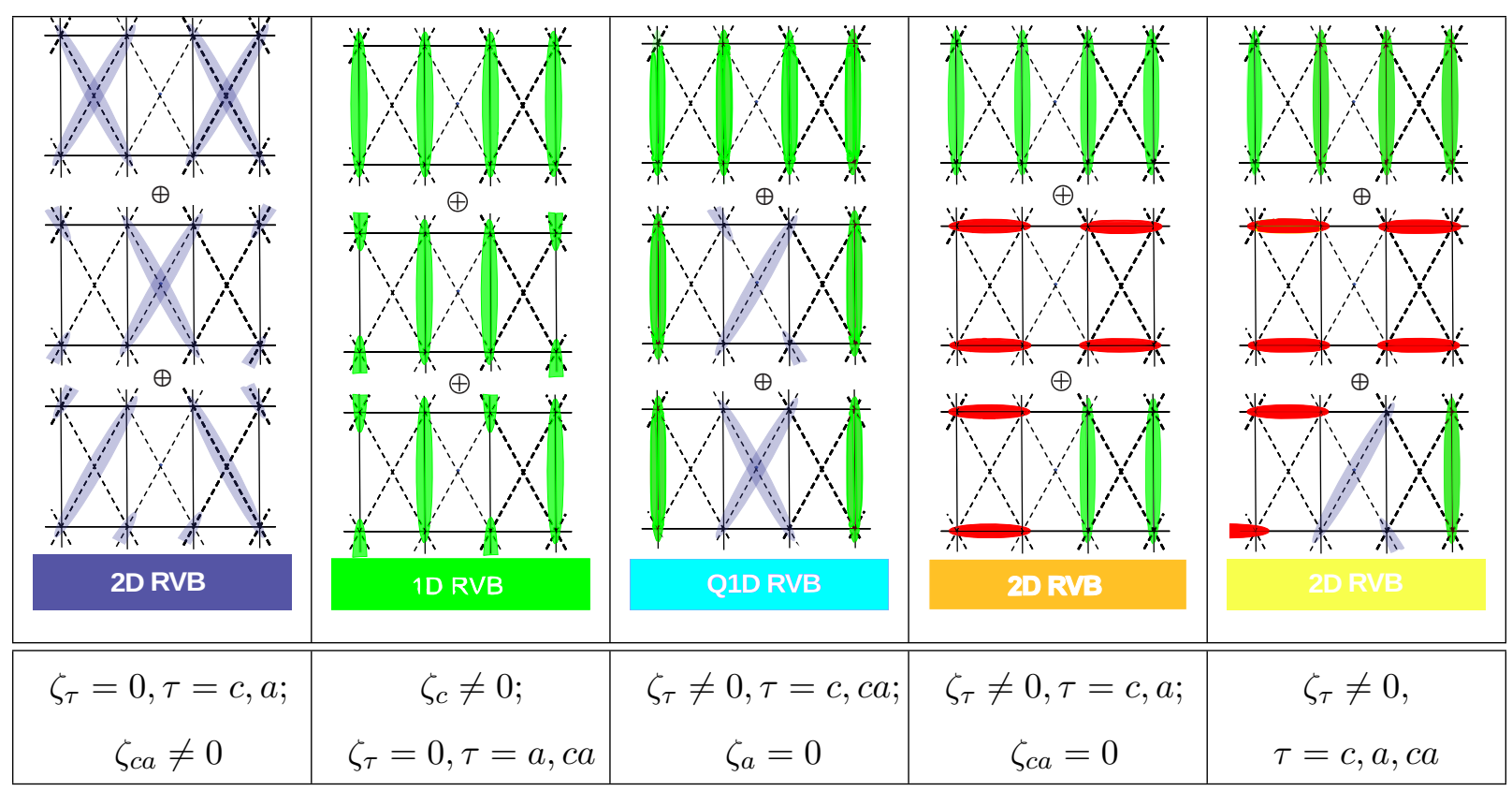

Figure 11

A. Tchougréeff, E. Plekhanov,

R. Dronskowski

J. Comput. Chem. 

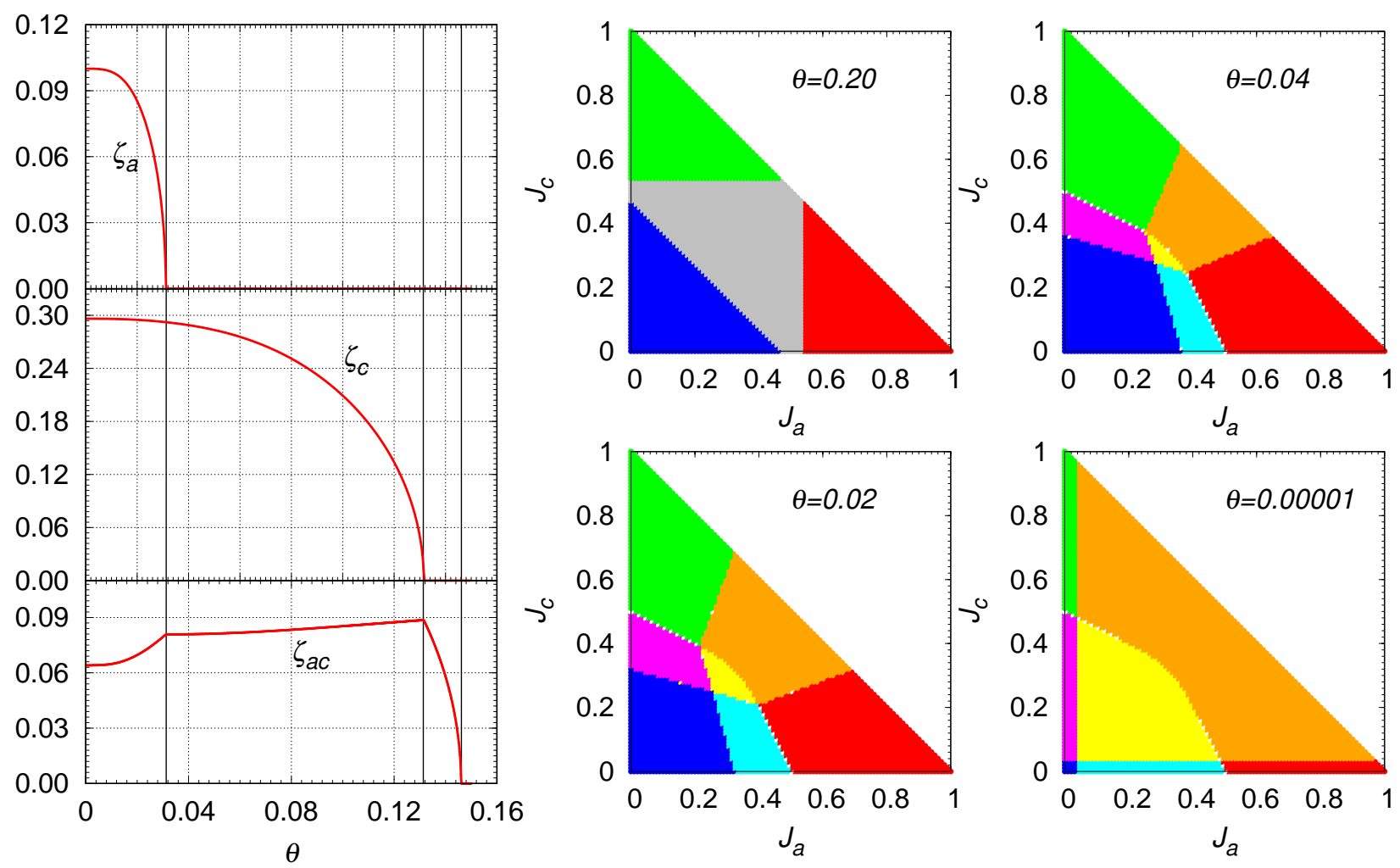

Figure 12

A. Tchougréeff, E. Plekhanov, R. Dronskowski

J. Comput. Chem. 


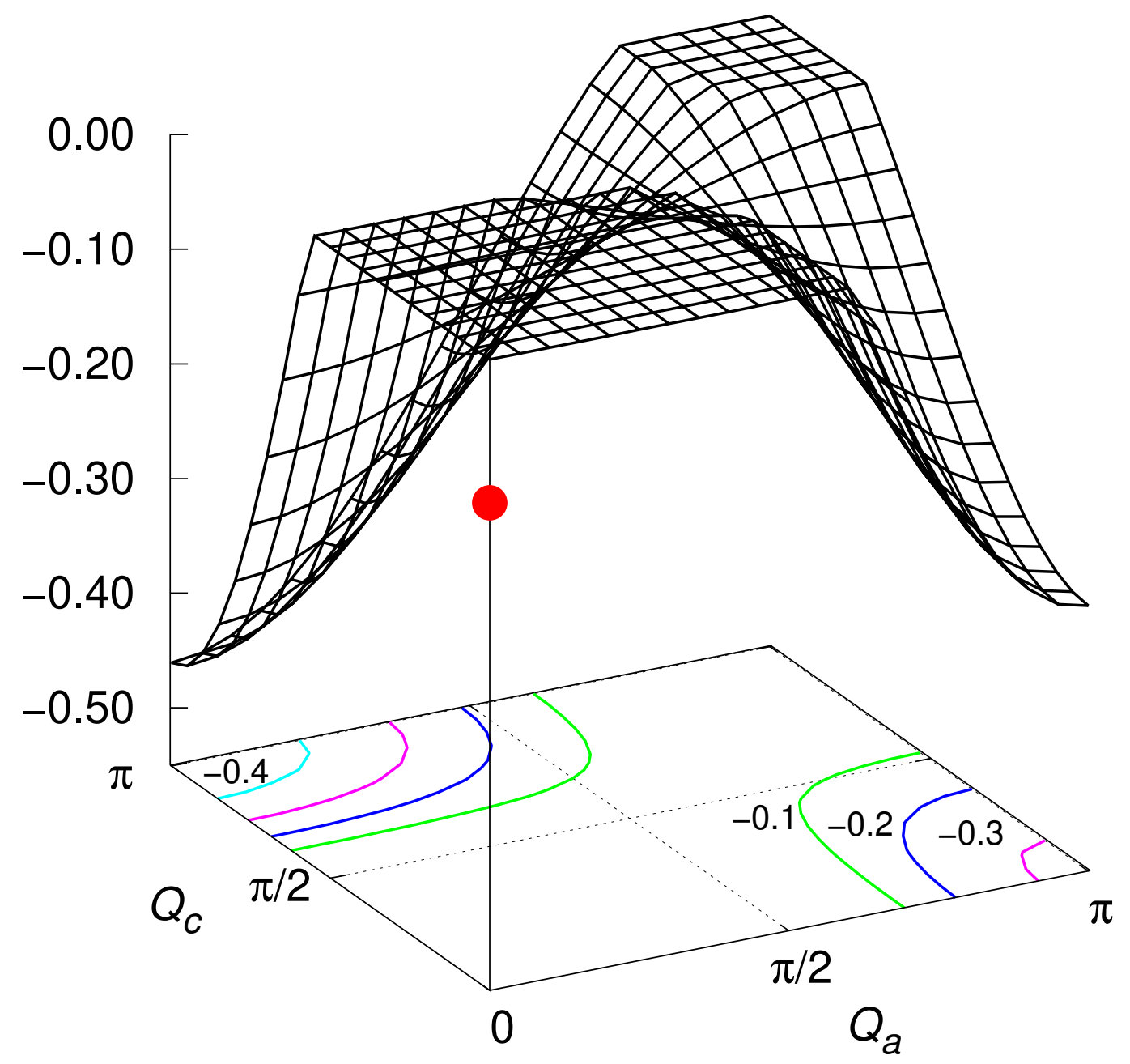

Figure 13

A. Tchougréeff, E. Plekhanov, R. Dronskowski

J. Comput. Chem. 


\begin{tabular}{|c|c|c|c|}
\hline & NM & FM $(\Theta \Phi)$ & FM(VASP) \\
\hline \hline$\Delta E_{\text {tot }}(\mathrm{eV})$ & 0 & -2.7 & -2.1 \\
\hline$\mu\left(\mu_{B}\right)$ & 0 & 3.4 & 3.1 \\
\hline
\end{tabular}

Table 1: Total energy gain $E_{t o t}$ and Fe magnetic moment comparison between $\Theta \Phi$ and VASP for ferromagnetic calculations in $\alpha$-Fe. 


\begin{tabular}{|c|c|c|c|c|c|}
\hline & NM & FM $(\Theta \Phi)$ & AFM $(\Theta \Phi)$ & FM(VASP) & AFM (VASP) \\
\hline \hline$\Delta E_{\text {tot }}(\mathrm{eV})$ & 0 & -2.64 & -2.60 & -2.32 & -2.24 \\
\hline$\mu\left(d\right.$-shell, $\left.\mu_{B}\right)$ & 0 & 3.24 & 3.10 & 3.24 & 3.10 \\
\hline
\end{tabular}

Table 2: Total energy gain $\Delta E_{t o t}$ and Fe $d$-shell magnetic moment comparison between $\Theta \Phi$ and VASP for ferromagnetic and anti-ferromagnetic $[\mathbf{Q}=\pi(0,1,1)]$ calculations in $\gamma$-Fe. 